\title{
Optimal ordering and pricing in a quick response system
}

\author{
Doğan A. Serel * \\ Faculty of Business Administration, Bilkent University, 06800 Bilkent, Ankara, Turkey
}

A R T I C L E I N F O

\section{Article history:}

Received 19 November 2007

Accepted 27 April 2009

Available online 13 May 2009

\section{Keywords:}

Inventory

Newsvendor

Price-dependent demand

Forecast update

Bayesian estimate

\begin{abstract}
A B S T R A C T
Quick response systems enable retailers to estimate customer demand more accurately, and improve stocking decisions for perishable products with uncertain demand. Retailers place separate orders for a product at two different times before the selling season. Following the initial order, additional market information is obtained, and the second-order amount is decided based on an improved demand forecast. In some cases, purchase cost associated with the second order is uncertain, and demand for the product during the season depends on the selling price. We present a solution procedure for finding the optimal order quantity and selling price in this setting. We also study the case where any desired portion of the initial order can be cancelled after updating the demand forecast. In the numerical study, the optimal price is observed to be relatively insensitive to changes in demand variability.
\end{abstract}

(c) 2009 Elsevier B.V. All rights reserved.

\section{Introduction}

Forecasting demand for products as accurately as possible is crucially important for maintaining the profitability of retail businesses. Advances in information technology have facilitated development of various new decision support tools that help the companies to control inventory levels in a cost-efficient manner.

Quick response systems in fashion apparel industry aim at shortening manufacturing and distribution lead times by means of information technology such as Electronic Data Interchange (EDI) and Point of Sale scanner, by utilizing faster modes of transportation, and also by organizing the manufacturing operations around cellular manufacturing concepts (Fisher and Raman, 1996). Successful fashion retailers identify customer trends and changing preferences as they emerge, and using highly automated processes such as computer-aided design and computer-aided manufacturing (CAD/CAM),

\footnotetext{
* Tel.: +90312 2902415 ; fax: +903122664958

E-mail address: serel@bilkent.edu.tr
}

convert these ideas into concrete products within weeks (Christopher et al., 2004).

For managing inventories of style goods, quick response systems have become popular and have been used successfully by a number of major retailers in the US (Fisher and Raman, 1996). Style goods are essentially the seasonal products such as toys and fashion apparel. They have a long supply lead time relative to the length of the selling season, and hence the number of ordering opportunities is limited, generally to one or two. These products are not typically carried over into future selling periods. At the end of the selling season, inventory not sold during the regular season must be liquidated at a discounted price or otherwise disposed of. By updating the demand forecast as actual market data become available, quick response systems allow for adjusting the stock of a retail item as closely as possible to the optimal level. An implementation of quick response approach at Sport Obermeyer, a leading fashion skiwear designer and manufacturer, is described in Fisher and Raman (1996).

The appropriate inventory level for a product at the beginning of a selling season is determined by considering the tradeoff between the cost of unsold items at the end of the selling season and the cost of unsatisfied demand 
caused by stockouts during the season. In general, uncertainty in customer demand forecast is higher when there is more time until the selling season. As the selling season approaches, more information about the potential customer demand is acquired and demand forecasts become more reliable. For example, Bitran et al. (1986) report that demand for the products of a consumer electronics company occurs mainly in the last quarter of the year, and sales forecasts are made at the beginning of each quarter. For a particular product, the successive sales forecasts pertaining to the next Christmas season had a coefficient of variation of $1,0.5$, and 0.2 in January, April, and October, respectively.

A common practice utilizing information update in a quick response system is to split the traditional single purchase order into two lots. The first order has a longer lead time; the second order is placed closer to the selling season when the retailer is able to take advantage of the improved demand forecast in choosing the stocking level for the item. However, in some cases the unit purchase cost associated with the second order may be unknown when the first order is placed. Hence, uncertainties in both future demand and purchase cost must be taken into account when the retailer determines the size of the first order. While short lead times help the retailer make better decisions regarding the amount of item to stock (due to better demand forecast), possible increases in unit purchase cost is a discouraging factor in adopting an extreme strategy involving $100 \%$ postponement of the first order. An optimal sourcing strategy is likely to prescribe a combination of short lead time and long lead time alternatives (Fisher and Raman, 1996).

In some cases, late orders are always more costly than early orders. Consider the production problem faced by a semiconductor manufacturer. The long lead times associated with the low-cost, offshore production require that the firm sets the initial (offshore) production quantity before observing any demand (Cattani et al., 2008). After getting a demand signal, the manufacturer can produce additional units by utilizing its high-cost, domestic manufacturing capacity.

Yan et al. (2003) discuss a component procurement problem faced by a security system manufacturing company. A key part of security systems is the microcontroller which has a read-only memory (ROM) that can be installed in one of two ways. Micro-controller chip can be custom-made according to user-supplied data requirements during the wafer fabrication process. This option entails significant lead time. The second option is to purchase a micro-controller with a generic programmable ROM and add the program code after the chips are received. These micro-controllers with programmable ROMs can be procured in a shorter lead time but are twice as expensive as custom-made chips. The company uses both types of micro-controller chips to balance the tradeoff between uncertainty in demand for security systems, which depends on lead time, and unit purchase cost of micro-controllers.

A number of researchers have studied the optimal purchasing decisions in a quick response scheme by using newsvendor-like inventory models. It is commonly assumed in the literature that selling price is an exogenous and given parameter, independent of the demand for the product. While this assumption may be plausible when the firm is a price-taker, for some products it may be more appropriate to assume a negative relationship between demand and price, as in the case of fashion garments sold by a specialty retailer that owns a network of approximately 50 stores in the US (Federgruen and Heching, 1999). In this paper we treat both the order quantity and selling price as decision variables. We consider a retailer who has to decide how much to order at two different times prior to the selling season. The first order is based on an initial demand forecast. The second order is based on an improved demand forecast, but the purchase price in the second order is not known with certainty when the initial order is given. We consider that the retailer can estimate the average demand more accurately by collecting market information between the first and the second orders. The two-stage ordering problem that we explore in this paper is built upon the assumption of normal probability distribution for uncertain demand, which has also been employed by a number of researchers in the inventory literature. Our framework allows specification of error component in price-dependent demand according to two common approaches, namely additive and multiplicative errors. Determining the optimal solution in the problem with price-sensitive demand is not straightforward; we therefore propose a search procedure based on computation of the expected profit at different points in the search region.

We make a separate analysis of the retailer's ordering policy when it is possible to cancel the initial order fully or partially; we show that the expected profit is a concave function of the initial order amount. An algorithm for solving the retailer's ordering problem with order-cancellation flexibility is presented.

Results from our computational study indicate that higher demand variability leads to a decrease in the retailer's initial order size. We also observe that introduction of order-cancellation flexibility helps the retailer cope better with the negative impact of demand uncertainty, and the optimal price is fairly robust with changes in demand variability.

The paper is structured as follows. After reviewing the related literature, we present the key modeling assumptions in Section 3. In Section 4, we study the retailer's problem when selling price is fixed. The optimal ordering and pricing policy in the price-sensitive demand scenario is explored in Section 5. In Section 6, we look into the setting where the retailer has the ability to cancel any portion of the initial order after revising the demand forecast. Following numerical examples in Section 7, the concluding remarks are given in Section 8.

\section{Literature review}

For goods ordered periodically over time, a common approach followed by retailers for predicting the future demand is to adjust the previous forecast by taking into account the latest actual sales data available; time series 
methods such as exponential smoothing is widely used (e.g. Venkateswaran and Son, 2007). Various researchers have studied the benefits of demand information sharing in multi-echelon supply chains consisting of manufacturers, distributors and retailers (Wu and Edwin Cheng, 2008); by observing the customers' demand information directly, the manufacturers and distributors can reduce their inventory costs, and hence, the negative effect of demand variability on the supply chain is mitigated.

In style-goods inventory literature, the impact of quick response systems on the profits of the manufacturer and retailer has been investigated using multi-stage ordering models with demand forecast updating. A research stream has considered a setting where there are two selling periods and the retailer has a chance to adjust its inventory level at the beginning of the second period based on sales observed in the first period. Bradford and Sugrue (1990) study a two-period model in which a group of items have heterogeneous Poisson demands; for each item the parameter of the Poisson distribution is distributed according to a gamma distribution, resulting in a negative binomial distribution for the aggregate demand. An improved solution procedure for the problem discussed in Bradford and Sugrue (1990) is given by Lau and Lau (1999). Fisher and Raman (1996) look into the production commitment decisions of a manufacturer. An initial commitment is made before receiving any customer orders; after observing initial demand, a second commitment is made in the second period. In each period, there are lower and upper limits on the production quantities of multiple products. Eppen and Iyer (1997) investigate backup agreements between a catalog retailer and manufacturers. A fixed fraction of the firm commitment is held as backup. After observing early demand, the catalog company can order additional units from this backup. Again in a two-period setting, Fisher et al. (2001) assume that the manufacturer delivers the updated order from the retailer after a significant lead time, and backorders for the first period can be filled from this replenishment. In Milner and Rosenblatt (2002), the buyer places initial orders for two periods at a specified unit price. At the end of the first period, the buyer can adjust the second period order by incurring order adjustment charges.

Our paper follows the other major research vein in which two orders given at two separate ordering instants prior to the selling season together constitute the inventory that will be available to the retailer to satisfy the demand in a single selling period. The demand forecast updating is based on information such as sales of related products, advance purchases, and other market observations. Gurnani and Tang (1999) explore a twostage ordering problem with uncertain purchase cost at the second-ordering instant. Yan et al. (2003) study a similar problem in which the known purchase cost is higher in the second order. As in Gurnani and Tang (1999), Choi et al. (2003) investigate the retailer's ordering decision in a problem with two ordering opportunities and uncertainty in the ordering cost at the second stage. But instead of defining a joint probability distribution of market information and demand, they use a Bayesian conjugate family (normal prior and posterior distribution) for the forecast update process. Choi et al. (2006) consider the case where the Bayesian updating procedure is applied to both the mean and variance of the demand distribution.

Choi et al. (2004) consider a problem where the retailer updates the demand forecast multiple times before the selling season; the purchase cost of the product increases and the forecast error decreases as time progresses. The problem is to determine the timing and size of the single order given by the retailer. Kim (2003) explores a similar problem in which the retailer is able to order not once but multiple times before the beginning of the selling period. Iyer and Bergen (1997) compare the effect of inventory decisions on the retailer and the manufacturer with or without using quick response. Donohue (2000) investigates channel-coordinating buy-back contracts in this kind of two-stage ordering systems. In a similar vein, Chen et al. (2006) investigate coordination of a system in which the manufacturer commits to a production quantity in the first stage. Choi and Chow (2008) extend the results of Iyer and Bergen (1997) to the case where the impact of a quick response system on both the mean and the variance of the profit distribution is taken into account. Ferguson et al. (2005) analyze a setting where a buyer can specify its order quantity based on either an initial or an updated demand forecast; in the early commitment case the buyer assumes all of the demand risk whereas in the delayed commitment case the risk is shared by the buyer and the supplier. Huang et al. (2005) study a two-stage purchase contract in which an order quantity is specified at stage 1 ; changing this order quantity after the forecast update incurs a fixed as well as a variable cost. Yan et al. (2008) describe an application of quick response in the textile industry in which a fabric manufacturing company places orders with yarn suppliers.

In general the retail price has been assumed to be fixed in the literature reviewed above. One of the exceptions is the two-period model of Petruzzi and Dada (2001) in which demand depends on the retail price, a decision variable. They assume the unsold stock at the end of the first period can be carried to the second period by incurring a transshipment cost. They determine optimal prices and order amounts for the two periods. Another paper built on price-dependent demand is Choi (2007) in which the retailer first determines the order quantity based on a tentative market price. Following the demand forecast update, based on the latest demand information and the available stock, the retailer sets the actual price to be charged during the single period.

Although the specific demand probability distributions and Bayesian updating mechanisms employed in the earlier literature may vary depending on the particular study, the common idea in all these papers is that reducing the variance of the demand forecast after collecting preliminary market data enables the retailer to choose a better stocking level, and hence reduces the expected inventory costs. A demand forecast update is also a critical element of our model, but unlike the traditional setup, we consider the retailer's two-stage ordering problem when demand is price sensitive, and offer a practical approach to calculate the retailer's expected profit. 


\section{Model}

We consider a retailer who needs to stock the appropriate amount of a style good with uncertain demand prior to the selling season. The first order must be given at time 1, before the retailer can gather additional information about the potential demand; the unit wholesale price at time 1 is $c_{1}$. The retailer's second order is given at time 2 after the forecast revision; the unit wholesale price $c_{2}$ at time 2 is assumed to have a discrete probability distribution. We use $c_{2 i}$ to denote the wholesale price in state $i$ at time $2, i=1, \ldots, n$, and the probability of state $i$ is denoted by $w_{i}$. It is possible that the observed wholesale price at time 2 can be lower than that at time 1 . The retailer sells the item during the regular season at unit price $p$. We first consider $p$ as given, and investigate the optimal ordering policy. In a later section, we explore the joint optimization of price and order quantity under price-sensitive demand. The unit holding cost (physical carrying cost minus salvage value) associated with leftover items is denoted by $h$. Each unsold unit at the end of the selling season results in a cost of $h$, which can be negative if the salvage value is greater than the physical carrying cost.

For modeling the demand probability distribution and Bayesian updating of the distribution parameter, we adopt the approach used by Iyer and Bergen (1997) and by Choi et al. (2003). At time 1, customer demand forecast is a normal probability distribution with unknown mean $m$ and known variance $\sigma_{1}^{2}$. There is uncertainty regarding the mean of the distribution, $m$. We assume $m$ is also normally distributed with mean $\mu_{1}$ and variance $d_{1}$. A higher value for $d_{1}$ indicates that the retailer is less informed about $m$. Hence, the unconditional probability distribution of demand at time 1 is a normal distribution with mean $\mu_{1}$ and variance $\sigma_{1}^{2}+d_{1}$. The normal probability distribution for demand has been commonly used in the inventory literature; in practice, the vast majority of commercial inventory systems assume normal demand distribution (Nahmias, 1994). The normal distribution is frequently assumed in the newsvendor model because of the central limit theorem, and also because of its entropy-maximizing property (Perakis and Roels, 2008). Among all distributions with given mean and variance, the normal distribution maximizes entropy, which is a measure of the amount of uncertainty. Thus, the normal distribution is a "robust" distribution when the decision maker knows only the mean and variance of the distribution.

The retailer orders $Q_{1}$ units at time 1 . Between time 1 and time 2, market information is gathered and translated into an observation about demand, say $x$. In practice, the "new market information" is obtained through trade shows, marketing research and early order commitments (Donohue, 2000). Some Internet retailers sell products such as movies, music CDs and books at a discounted price before they are released to the broader market; these pre-committed orders by customers are used to improve the market demand forecast for the product (Tang et al., 2004). The observed sales of related items can be used to decrease forecast error for the product (Iyer and Bergen, 1997). In fashion apparel industry, the information on sales of clothes with a particular color (e.g. red) is useful for predicting the demand of other clothes sharing the same color (Choi et al., 2003). Two different CD albums by the same artist is another example for products with correlated sales (Choi et al., 2006).

The distribution of the location parameter $m$ is updated based on $x$. By Bayesian theory, the posterior distribution of $m$ will be normal with mean $\left[\left(1 / \sigma_{1}^{2}\right) x+\left(1 / d_{1}\right) \mu_{1}\right] /-$ $\left[\left(1 / \sigma_{1}^{2}\right)+\left(1 / d_{1}\right)\right]$ and variance $\sigma_{1}^{2} d_{1} /\left(\sigma_{1}^{2}+d_{1}\right)$. The unconditional probability distribution of demand at time 2 will be a normal distribution with mean $\mu_{2}=\left(\mu_{1} \sigma_{1}^{2}+x d_{1}\right) /\left(\sigma_{1}^{2}+d_{1}\right)$, and variance $\sigma_{2}^{2}=\sigma_{1}^{2}\left[1+\left(d_{1} /\left(\sigma_{1}^{2}+d_{1}\right)\right)\right]$. Defining $d_{2}=\sigma_{1}^{2} d_{1} /$ $\left(\sigma_{1}^{2}+d_{1}\right)$, we have $\sigma_{2}^{2}=\sigma_{1}^{2}+d_{2}$.

Based on the updated distribution for demand, and the observed purchase cost $c_{2 i}$, the retailer orders $Q_{2 i}$ units at time 2. Thus, the total stock available at the beginning of the selling season will be $Q_{1}+Q_{2 i}$.

\section{Optimal ordering policy when selling price is fixed}

The retailer's optimal order quantity $Q_{1}$ at time 1 can be found in two steps using backward dynamic programming. First, for a given $Q_{1}$ and the demand observation $x$, we derive the expression defining the optimal order quantity at time 2. Second, by substituting the expression for optimal $Q_{2 i}$ in the retailer's objective function at time 1 , we determine the optimal $Q_{1}$.

The retailer's expected profit at time 2 as a function of $Q_{1}$ and $Q_{2 i}, B_{2 i}$ is given

$B_{2 i}\left(Q_{1}, Q_{2 i}\right)=p E\left[\min \left(Q_{1}+Q_{2 i}, Y\right)\right]-h E\left[Q_{1}+Q_{2 i}-Y\right]^{+}-c_{2 i} Q_{2 i}$,

where $Y$ stands for the random demand, the expectations are with respect to the probability distribution of demand at time 2, i.e., a normal distribution with mean $\mu_{2}$ and variance $\sigma_{2}^{2}$, and $(K)^{+} \equiv \max (K, 0)$. Note that $Q_{2 i}$, the order quantity in state $i$, is selected after observing the random purchase cost $c_{2 i}$ at time 2 . Setting the derivative of $B_{2 i}$ to zero, we obtain the optimal $Q_{2 i}, Q_{2 i}^{*}$ :

$Q_{2 i}^{*}=\max \left\{0, \mu_{2}+\left(d_{2}+\sigma_{1}^{2}\right)^{0.5} \Phi^{-1}\left(s_{i}\right)-Q_{1}\right\}$,

where $\Phi^{-1}(\cdot)$ is the inverse cumulative distribution function (cdf) of standard normal distribution and $s_{i}$ is the standard critical fractile solution of the newsvendor problem, i.e.,

$s_{i}=\left(p-c_{2 i}\right) /(p+h)$.

The retailer's expected profit at time 2 can be expressed as a sum of two parts, one conditional on $Q_{2 i}^{*}>0$, and the other conditional on $Q_{2 i}^{*}=0$. Let $t_{i}=\mu_{2}+\left(d_{2}+\sigma_{1}^{2}\right)^{0.5} \Phi^{-1}\left(s_{i}\right)$. Then, the retailer's expected profit when $Q_{2 i}^{*}>0, J_{1 i}\left(Q_{1}, \mu_{2}\right)$, is given by

$$
\begin{aligned}
J_{1 i}\left(Q_{1}, \mu_{2}\right)= & p E\left[\min \left(t_{i}, Y\right)\right]-h E\left[t_{i}-Y\right]^{+}-c_{2 i}\left(t_{i}-Q_{1}\right) \\
= & \left(p-c_{2 i}\right) \mu_{2}-\left(h+c_{2 i}\right)\left(d_{2}+\sigma_{1}^{2}\right)^{0.5} \Phi^{-1}\left(s_{i}\right) \\
& -(p+h)\left(d_{2}+\sigma_{1}^{2}\right)^{0.5} \Psi\left(\Phi^{-1}\left(s_{i}\right)\right)+c_{2 i} Q_{1},
\end{aligned}
$$

where $\Psi(u)=\int_{u}^{\infty}(z-u) \phi(z) d z$ is the unit loss function for the standard normal distribution, and $\phi(z)$ is the standard normal probability density function (pdf). If the updated mean demand at time 2 is less than $Q_{1}-\left(d_{2}+\sigma_{1}^{2}\right)^{0.5} \Phi^{-1}\left(s_{i}\right)$, 
i.e., if $t_{i}<Q_{1}$, no order will be placed at time 2 , and the retailer's expected profit, $J_{2}\left(Q_{1}, \mu_{2}\right)$, will be

$$
\begin{aligned}
J_{2}\left(Q_{1}, \mu_{2}\right)= & p E\left[\min \left(Q_{1}, Y\right)\right]-h E\left[Q_{1}-Y\right]^{+} \\
= & p \mu_{2}+h\left(\mu_{2}-Q_{1}\right)-(p+h)\left(d_{2}+\sigma_{1}^{2}\right)^{0.5} \\
& \times \Psi\left[\left(Q_{1}-\mu_{2}\right) /\left(d_{2}+\sigma_{1}^{2}\right)^{0.5}\right] .
\end{aligned}
$$

By taking expectation of $J_{1 i}$ and $J_{2}$ over the probability distributions of $\mu_{2}$ and the unit purchase cost at time 2, we can write the retailer's expected profit at time $1, B_{1}$, as

$$
\begin{aligned}
B_{1}\left(Q_{1}\right)= & \sum_{i=1}^{n} w_{i} \int_{-\infty}^{\infty} B_{2 i}\left(Q_{1}, Q_{2 i}^{*}\right) g\left(\mu_{2}\right) d \mu_{2}-c_{1} Q_{1} \\
= & \sum_{i=1}^{n} w_{i}\left\{\int_{Q_{1}-\left(d_{2}+\sigma_{1}^{2}\right)^{0.5} \Phi^{-1}\left(s_{i}\right)}^{\infty} J_{1 i}\left(Q_{1}, \mu_{2}\right) g\left(\mu_{2}\right) \times d \mu_{2}\right. \\
& \left.+\int_{-\infty}^{Q_{1}-\left(d_{2}+\sigma_{1}^{2}\right)^{0.5} \Phi^{-1}\left(s_{i}\right)} J_{2}\left(Q_{1}, \mu_{2}\right) g\left(\mu_{2}\right) d \mu_{2}\right\}-c_{1} Q_{1},
\end{aligned}
$$

where $g\left(\mu_{2}\right)$ is the pdf of $\mu_{2}$, viz., a normal distribution with mean $\mu_{1}$ and variance $\sigma^{2}=\left[d_{1}^{2} /\left(d_{1}+\sigma_{1}^{2}\right)\right] . B_{1}\left(Q_{1}\right)$ is concave in $Q_{1}$, so the optimal $Q_{1}$, if positive, satisfies the first-order condition (Choi et al., 2003):

$$
\begin{aligned}
\partial B_{1} / \partial Q_{1}= & \sum_{i=1}^{n} w_{i}\left\{\left(p-c_{2 i}\right) \Phi\left(\varepsilon_{i}\right)+\left(c_{2 i}-c_{1}\right)\right. \\
& \left.-(p+h) \int_{-\infty}^{\kappa_{i}} \Phi\left(\frac{Q_{1}-\mu_{1}-\gamma \sigma}{\sqrt{d_{2}+\sigma_{1}^{2}}}\right) \phi(\gamma) d \gamma\right\}=0,
\end{aligned}
$$

where $\kappa_{i}=Q_{1}-\left(d_{2}+\sigma_{1}^{2}\right)^{0.5} \Phi^{-1}\left(s_{i}\right), \quad \gamma=\left(\mu_{2}-\mu_{1}\right) / \sigma$, and $\varepsilon_{i}=\left[Q_{1}-\left(d_{2}+\sigma_{1}^{2}\right)^{0.5} \Phi^{-1}\left(s_{i}\right)-\mu_{1}\right] / \sigma$.

To express (2) in a more compact way, we can evaluate the integral term by using

$$
\begin{aligned}
& \int_{-\infty}^{z} \Phi\left(\frac{Q_{1}-\mu_{1}-\gamma \sigma}{\sqrt{d_{2}+\sigma_{1}^{2}}}\right) \phi(\gamma) d \gamma \\
& =B N\left(\frac{\alpha}{\sqrt{1+\beta^{2}}}, z ; \quad \rho=-\frac{\beta}{\sqrt{1+\beta^{2}}}\right),
\end{aligned}
$$

where $B N(\cdot)$ is the standard bivariate normal cdf, i.e.,

$$
\begin{aligned}
B N(h, k ; \rho)= & \frac{1}{2 \pi \sqrt{1-\rho^{2}}} \\
& \times \int_{-\infty}^{k} \int_{-\infty}^{h} \exp \left[-\left(\frac{x^{2}-2 \rho x y+y^{2}}{2\left(1-\rho^{2}\right)}\right)\right] d x d y,
\end{aligned}
$$

$\alpha=\left(Q_{1}-\mu_{1}\right) / \sqrt{d_{2}+\sigma_{1}^{2}}$ and $\beta=-\left(\sigma / \sqrt{d_{2}+\sigma_{1}^{2}}\right)$ (Owen, 1980). Thus, (2) can be computed easily by substituting (3) in (2). In Appendix A, we present a method for calculating the retailer's expected profit function $B_{1}\left(Q_{1}\right)$.
The method is based on transforming complicated expressions to more tractable ones that can be evaluated using already existing standard algorithms for the normal probability distribution.

\section{Optimal policy under price-dependent demand}

While the single-period inventory problem with a fixed selling price has been a useful building block for many previous studies, the assumption of a constant selling price may be too restrictive in certain situations, and it is more appropriate to treat price as a controllable variable (e.g. Ray et al., 2005; Teng et al., 2005; Serel, 2008). The extension of the standard newsvendor problem to the case where demand is negatively related to the selling price has received considerable interest from researchers in the operations management field (e.g. Lau and Lau, 1988). For a recent review of the literature, see Petruzzi and Dada (1999). On the other hand, only a few papers have considered the case of price-sensitive demand in a quick response framework with demand forecast updating (Petruzzi and Dada, 2001; Choi, 2007). Since pricesensitive demand is a relevant issue in a wide variety of practical settings, we extend the two-stage model with a constant selling price to the case where both price and order quantities are decision variables.

We consider the additive uncertainty approach such that the random demand $Y$ in the selling season is

$$
Y=y(p)+\zeta,
$$

where $y(p)$ is the deterministic part of the demand and $\zeta$ is a random variable with mean $\mu_{e}$ and variance $\sigma_{e}^{2}$. The additive error model assumes that the variance of demand does not change as price changes. This assumption is also empirically supported for certain products; based on data collected from a retailer of high-end women's apparel in the US, Federgruen and Heching (1999) report that the standard deviation of nation-wide weekly sales is independent of selling price. Regarding $y(p)$, we assume that the expected demand is linearly related to the selling price, i.e., $y(p)=a-b p$ where $a$ and $b$ are known parameters $(a, b>0)$. The selling price $p$ is set by the retailer after observing the random $\operatorname{cost} c_{2 i}$ at time 2 . The order of decisions by the retailer is shown in Fig. 1. After observing the state of the world at time 2, the optimal price associated with that state is selected for the season.

In general, there are various ways to incorporate the additional market information collected by the retailer between time 1 and time 2 into a model involving

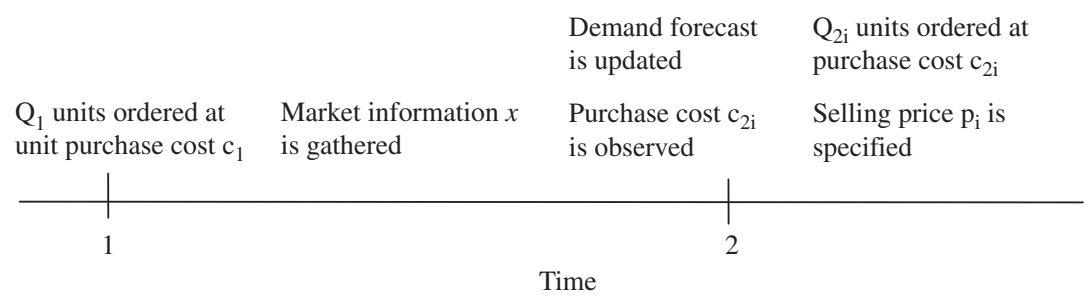

Fig. 1. The sequence of decisions over time line. 
price-dependent demand. For example, it can be assumed the parameter $a$ or $b$ is not known with certainty, and the new information is used to update this unknown parameter. Alternatively, it can be assumed that $a$ and $b$ are known, and the additional information is used to improve the estimates of the parameters of the error distribution. We can relate this latter approach to the core model in the earlier section more directly and easily. The model in Section 4 corresponds to the special case $a=b=0$ of the model discussed in this section.

In this section we will assume that all parameters except $\mu_{e}$ are known, and the Bayesian estimation procedure is applied to the mean of the distribution of $\zeta$ only. Thus, the expected demand is $a-b p+\mu_{e}$, the deterministic part of the demand $y(p)=a-b p$ is assumed to be known, and there is a prior probability distribution on the unknown error mean $\mu_{e}$ at time 1 . The error is normally distributed with unknown mean $\mu_{e}$ and known variance $\sigma_{1}^{2}$, and $\mu_{e}$ is normally distributed with mean $\mu_{1}$ and variance $d_{1}$ at time 1. The market information will be used to obtain the posterior distribution of $\mu_{e}$ and the predictive distribution of the additive error $\zeta$ at time 2 . With this assumption, the particular method developed earlier for solving the retailer's problem can be smoothly integrated with the solution procedure for the joint pricing-inventory problem that we explore in this section. We remark that in Petruzzi and Dada (2001) the demand forecast update is based on the observed additive error, and as in our model, the deterministic part of the demand $y(p)$ is assumed to be known.

\subsection{Deterministic demand}

For ease of exposition and as a precursor, we start by considering the deterministic demand case where $\mu_{\mathrm{e}}$ is assumed to be known and $\sigma_{e}^{2}=0$. Demand as a function of price is given by

$Y=a-b p+\mu_{e}$.

Although there is no forecast update when demand is deterministic, due to uncertainty in the purchase cost at time 2, we continue to have a two-stage problem. Since the retailer has a second purchase opportunity at time 2 , the optimal policy should specify the purchase quantity at time $1 Q_{1}^{*}$ as well as the optimal price $p_{i}^{*}$ and purchase quantity $Q_{2 i}^{*}$ in state $i$ at time $2, i=1, \ldots, n$. For a given initial order $Q_{1}$, the retailer will order additional units if the purchase cost at time $2 c_{2 i}$ is low enough, and it will not order new units if $c_{2 i}$ is relatively high. Suppose it is optimal for the retailer to order additional units in state $i$ at time 2 . Given that $Q_{1}$ units were ordered at time 1 , the retailer who is deciding the additional order amount in state $i$ at time 2 will try to maximize

$R P^{(i)}=p^{(i)}\left(a-b p^{(i)}+\mu_{e}\right)-c_{2 i}\left(a-b p^{(i)}+\mu_{e}-Q_{1}\right)-c_{1} Q_{1}$,

where $p^{(i)}$ is the selling price chosen by the retailer in state $i$ for a given $Q_{1}$. The price $p_{i}$ maximizing (4) is given by

$p_{i}=\left(a+b c_{2 i}+\mu_{e}\right) / 2 b$.

Now suppose it is not optimal to order additional units in state $i$ at time 2 . As long as $Q_{1}$ is less than the single-stage profit maximizing order quantity $Q_{f}$ (defined below), the retailer will select the price such that the demand will be equal to the available stock, i.e., $Q_{1}$. The price that leads to this outcome is

$p^{i}=\left(a+\mu_{e}-Q_{1}\right) / b$.

We note that the optimal initial order $Q_{1}^{*}$ is always less than or equal to $Q_{f}$. Thus, for a given $Q_{1}$, we can place the $n$ states at time 2 into two different groups, $S_{1}$ and $S_{2}$. When in a state in set $S_{1}$, the retailer will issue a positive order $\left(Q_{2 i}>0\right)$, set $p^{(i)}=p_{i}$, and bring the total inventory to

$Y^{(i)}=a-b p_{i}+\mu_{e}$.

When in a state in set $S_{2}$, the retailer will not order additional units $\left(Q_{2 i}=0\right)$, and set $p^{(i)}=p^{i}$. For the states in set $S_{1}$, it should hold that $Y^{(i)}>Q_{1}$, and for the states in set $S_{2}$ we have $Y^{(i)} \leq Q_{1}$. Hence, for a given $Q_{1}$ the retailer's profit function $R P\left(Q_{1}\right)$ can be written as

$$
\begin{aligned}
R P\left(Q_{1}\right)= & -c_{1} Q_{1}+\sum_{i \in S_{1}} w_{i}\left[p_{i} Y^{(i)}-c_{2 i}\left(Y^{(i)}-Q_{1}\right)\right] \\
& +\sum_{i \in S_{2}} w_{i}\left(\frac{a+\mu_{e}-Q_{1}}{b}\right) Q_{1},
\end{aligned}
$$

where $S_{1}=\left\{i: Y^{(i)}>Q_{1}\right\}$, and $S_{2}=\left\{i: \quad Y^{(i)} \leq Q_{1}\right\}$. To act optimally in state $i$ at time 2 , the retailer compares $Q_{1}$ with the state-dependent threshold $Y^{(i)}$, and if $Q_{1}<Y^{(i)}$, $Y^{(i)}-Q_{1}$ units are ordered; if $Q_{1} \geq Y^{(i)}$ no additional order is made and the selling price is set to $p^{i}$.

As $Q_{1}$ increases from 0 , the number of states in set $S_{1}$ decreases, and correspondingly the set $S_{2}$ is enlarged. Although at first $R P\left(Q_{1}\right)$ may appear discontinuous in $Q_{1}$, it is actually a continuous function of $Q_{1}$. To see this, consider a state $j$ such that $j \in S_{1}$ for $Q_{1}=Y^{(j)-}$, and $j \in S_{2}$ for $Q_{1}=Y^{(j)+}$, where $Y^{(j)-}=\lim _{\varepsilon \rightarrow 0} Y^{(j)}-\varepsilon, Y^{(j)+}=\lim _{\varepsilon \rightarrow}$ ${ }_{0} Y^{(j)}+\varepsilon$, and $\varepsilon>0$. Thus we consider the neighborhood of $Q_{1}=Y^{(j)}$ where state $j$ moves from set $S_{1}$ to set $S_{2}$. Then as $\varepsilon \rightarrow 0$, we have

$w_{j}\left[p_{j} Y^{(j)}-c_{2 j}\left(Y^{(j)}-Y^{(j)-}\right)\right]=w_{j}\left[\left(\frac{a+\mu_{e}-Y^{(j)+}}{b}\right) Y^{(j)+}\right]$,

implying that $R P\left(Q_{1}\right)$ is continuous. Since, from (8),

$\frac{\partial R P\left(Q_{1}\right)}{\partial Q_{1}}=-c_{1}+\sum_{i \in S_{1}} w_{i} c_{2 i}+\sum_{i \in S_{2}} \frac{w_{i}\left(a+\mu_{e}-2 Q_{1}\right)}{b}$,

$\frac{\partial^{2} R P\left(Q_{1}\right)}{\partial Q_{1}^{2}}=\sum_{i \in S_{2}} \frac{-2 w_{i}}{b} \leq 0$,

$R P\left(Q_{1}\right)$ is concave in $Q_{1}$. Using the first-order condition $\partial R P\left(Q_{1}\right) / \partial Q_{1}=0$, if set $S_{2}$ is not empty at the optimal solution, the optimal $Q_{1}$ satisfies

$Q_{1}^{*}=\frac{-b c_{1}+b \sum_{i \in S_{1}} w_{i} c_{2 i}+\left(a+\mu_{e}\right) \sum_{i \in S_{2}} w_{i}}{2 \sum_{i \in S_{2}} w_{i}}$

Note that if we know which states are included in sets $S_{1}$ and $S_{2}$ in the optimal solution, $Q_{1}^{*}$ can be determined using (11). From (9), we have

$\left.\frac{\partial R P\left(Q_{1}\right)}{\partial Q_{1}}\right|_{Q_{1}=0}=-c_{1}+\sum_{i=1}^{n} w_{i} c_{2 i}$, 
since when $Q_{1}=0$, set $S_{1}$ contains all states $i=1, \ldots, n$. Thus, if $c_{1}>\sum_{i=1}^{n}{ }_{1} W_{i} c_{2 i}, \partial R P\left(Q_{1}\right) / \partial Q_{1}<0$ at $Q_{1}=0$. Since $R P\left(Q_{1}\right)$ is concave in $Q_{1}$, this implies that $\partial R P\left(Q_{1}\right) / \partial Q_{1}<0$ for $Q_{1}>0$. Hence, when we have the special case where $c_{1}>\sum_{i=1}^{n} w_{i} c_{2 i}$, the optimal solution is $Q_{1}^{*}=0, Q_{2 i}^{*}=Y^{(i)}$ and $p_{i}^{*}=p_{i}, i=1, \ldots, n$. The retailer's optimal profit is

$R P\left(Q_{1}=0\right)=\sum_{i=1}^{n} w_{i}\left(p_{i}-c_{2 i}\right) Y^{(i)}$.

Using $p_{i}-c_{2 i}=\left(a+\mu_{e}\right) / b-p_{i}$, and after some algebra, we obtain

$R P(0)=\sum_{i=1}^{n} \frac{w_{i}\left(a+\mu_{e}-b c_{2 i}\right)^{2}}{4 b}$.

To find an upper bound on the optimal initial order quantity, we consider the single-stage problem in which purchasing is allowed at time 1 only. A single-stage problem is equivalent to a two-stage problem with $n$ additional constraints $Q_{2 i}=0, i=1, \ldots, n$. The retailer's profit in the single-stage problem would be

$R P^{1}=\left(p-c_{1}\right)\left(a-b p+\mu_{e}\right)$

and, using the first-order condition, the optimal price would be

$p_{f}=\left(a+b c_{1}+\mu_{e}\right) / 2 b$.

The profit $\mathrm{RP}^{1}$ evaluated at $p=p_{f}$ can be regarded as a lower bound for the retailer's optimal profit $R P\left(Q_{1}^{*}\right)$ since it ignores the possibility of sourcing some units at a cost less than $c_{1}$ at time 2 . The demand at $p=p_{f}, a-b p_{f}+\mu_{\mathrm{e}}$, is an upper bound on the optimal $Q_{1}$ since in the case where the retailer can also order at time 2 , the optimal $Q_{1}$ will not exceed this upper bound. Let the upper bound on $Q_{1}$ be defined by

$Q_{f}=a-b p_{f}+\mu_{e}$.

Note that when $c_{1} \leq \min \left(c_{21}, c_{22}, \ldots, c_{2 n}\right)$, the optimal solution $Q_{1}^{*}=Q_{f}$ and $Q_{2 i}^{*}=0, i=1, \ldots, n$.

We propose Algorithm 1 in order to determine the retailer's optimal two-stage policy when demand is deterministic and price sensitive. In this algorithm, we first identify the optimal price $p^{(i)}$ and the optimal additional order $Q_{2 i}$ in state $i$ at time 2 given $Q_{1}$, and then find the optimal $Q_{1}$ by comparing expected profit values associated with different $Q_{1}$ values. When searching for the optimal $Q_{1}$, we use the increment $\Delta Q$ to discretize the search region, $\Delta Q=Q_{f} /(k-1)$ where $k$ is the maximum number of iterations allowed in Algorithm $1 ; k$ (an integer greater than or equal to 2 ) should be specified by the user.

Algorithm 1 (Optimal solution-deterministic demand). Step 0: Set $Q_{1}=0$, maxprofit $=0$.

Step 1: For each state $i$, compare $Y^{(i)}$ and $Q_{1}$. If $Y^{(i)}>Q_{1}$, set $p^{(i)}=p_{i}$, and $Q_{2 i}=Y^{(i)}-Q_{1}$. If $Y^{(i)} \leq Q_{1}$, then compare $Q_{1}$ and $Q_{f}$. If $Q_{1}=Q_{f}$, set $p^{(i)}=p_{f}$, and $Q_{2 i}=0$. If $Q_{1}<Q_{f}$, set $p^{(i)}=p^{i}$, and $Q_{2 i}=0$.

Step 2: Calculate the retailer's expected profit given $Q_{1}$ from (8). If $R P\left(Q_{1}\right)>$ maxprofit, set maxprofit $=R P\left(Q_{1}\right)$, $Q_{1}^{*}=Q_{1}, p_{i}^{*}=p^{(i)}, Q_{2 i}^{*}=Q_{2 i}$. If $R P\left(Q_{1}\right) \leq$ maxprofit, stop.
Step 3: Set $Q_{1}=Q_{1}+\Delta Q$. If $Q_{1} \leq Q_{f}$, repeat Steps 1 and 2 . Otherwise, stop.

The retailer's maximal expected profit is given by maxprofit at the end of Algorithm 1. In Step $1, Q_{2 i}=0$ if $Y^{(i)} \leq Q_{1}$ since in this case the marginal benefit of ordering an extra unit at time 2 is less than the marginal cost of an extra unit $c_{2 i}$. If $Q_{1}=Q_{f}$, the retailer is able to set the capacity-unconstrained optimal price $p_{f}$, and maximizes his expected profit by selling $Q_{f}$ units. On the other hand, if $Q_{1}<Q_{f}$, to maximize the profit, the retailer chooses the price $p^{i}$ that will liquidate all the available stock. If the optimal $Q_{1}$ is not zero, because of the concavity of $R P\left(Q_{1}\right)$, as $Q_{1}$ increases $R P\left(Q_{1}\right)$ first increases and then decreases. In Step 2, the search is finished when the profit is less than the profit at the previous iteration; at this point it is not necessary to consider higher values of $Q_{1}$ since the retailer's profit $R P\left(Q_{1}\right)$ will decrease as we continue to increase $Q_{1}$.

\subsection{Stochastic demand}

The joint inventory and pricing problem under stochastic demand can be solved by using an approach similar to that in the deterministic demand case. As in the deterministic demand case, we consider that an appropriate selling price needs to be specified in each state $i$ at time $2, i=1, \ldots, n$. The optimal price to charge in each state $p_{i}^{*}$ does not have an easily computed closed-form expression when demand is stochastic. Hence, more computational effort is required when demand is not deterministic.

The expected demand for the product equals the deterministic part $(a-b p)$ plus the estimated mean of the error term. To calculate the retailer's expected profit at time 1 , we combine the profit resulting from the deterministic part of demand $(a-b p)$, and the profit resulting from the inventory $Q_{1}-(a-b p)$. In the new model, $\mu_{1}$ corresponds to the estimated mean of the error distribution at time 1 . Basically, at time 2 the retailer will use the market information to update the estimate of the mean of the error distribution.

We now outline the procedure for finding the optimal solution. Suppose $Q_{1}$ is given and there is only one state $i$ at time 2 with purchase cost $c_{2 i}$, i.e., we consider a twostage problem with a single state at time 2 . Although price is a continuous variable, we find a nearly optimal price by considering a finite number of values in a search region which is known to contain the optimal price. During the search the expected profit for a particular price, denoted as $E P\left(Q_{1}, p, i\right)$, is computed using the expression for $B_{1}\left(Q_{1}\right)$ given by (1). Now suppose there is a single state of the world at time 2 , say state $j$ with purchase $\operatorname{cost} c_{2 j}, j \neq i$. For a given $Q_{1}$ the optimal selling price in this state can be found in the same manner as in state $i$. Using the same $Q_{1}$ value for each state $i, i=1, \ldots, n$, we conduct this search for optimal price and also record the optimal expected profit at time 1 , say $\operatorname{profit}\left(Q_{1}, i\right)$, at each of the $n$ iterations. Combining the solutions found for $n$ problems with a single state at time 2 , we can specify the optimal solution (set of optimal prices) for the problem with $n$ states given 
a specific $Q_{1}$ value. The weighted sum of $\operatorname{profit}\left(Q_{1}, i\right)$ using the weight $w_{i}$ for state $i$ yields the expected profit at time 1 for a given $Q_{1}$ in the two-stage problem involving $n$ states at time 2 . The expected profit at time 1 for a given $Q_{1}$ is referred to as $B\left(Q_{1}\right)$ in the algorithm used. Repeating the procedure described above we can compute the expected profit at time 1 for different $Q_{1}$ values. Based on these computations, the optimal $Q_{1}$ is found by a grid search over a region which is known to contain the optimal $Q_{1}$.

In order to find the optimal solution, we modify Algorithm 1 by adding an inner loop that searches the best price given $Q_{1}$ and the supply cost at time 2 . To define this loop, the lowest and highest feasible prices, $p^{1}$ and $p^{u}$, respectively, should be specified by the user.

Algorithm 2 presented below can be used to determine the optimal inventory and pricing decisions under stochastic demand. The main idea is first to find the optimal price and profit for each $Q_{1}$ value in the search space, and then determine the optimal $Q_{1}$ by using those profits computed. The algorithm finds the best price in each state at time 2 for a given $Q_{1}$. Then the retailer's expected profit for a given $Q_{1}$ is computed based on the optimal actions planned for each state at time 2 . The optimal $Q_{1}$ is determined by comparing those expected profits calculated for different $Q_{1}$ values. The optimal price in state $i$ at time 2 for a given $Q_{1}$ is denoted by $p_{i}^{\prime}$ in Algorithm 2 whereas $p_{i}^{*}$ indicates the optimal price in state $i$ associated with the optimal $Q_{1} . B\left(Q_{1}\right)$ in Algorithm 2 represents the retailer's expected profit at time 1 (including the profit associated with the deterministic part of demand).

The search regions for $p$ and $Q_{1}$ must be defined by the user. For example, the lower limit on price, $p^{1}$ can be set equal to the minimum possible unit purchase cost, i.e., $p^{1}=c_{\min }=\min \left(c_{1}, c_{21}, c_{22}, \ldots, c_{2 n}\right)$. The upper limit on price can be selected such that the expected demand (at time 1) for that price is zero, i.e., $p^{u}=\left(a+\mu_{1}\right) / b$. A loose upper limit on $Q_{1}, Q_{1}^{u}$, can be determined according to the newsvendor critical fractile solution at time 1 , i.e., $Q_{1}^{u}=a-b p^{1}+N^{-1}\left[\left(p^{u}-c_{\min }\right) /\left(p^{u}+h\right)\right]$, where $N^{-1}(\cdot)$ is the inverse cdf of a normally distributed random variable with mean $\mu_{1}$ and variance $\sigma_{1}^{2}+d_{1}$. The user also needs to input the increment $\Delta p$ used in the search for optimal $p$, $\Delta p=\left(p^{u}-p^{\mathrm{l}}\right) /(k-1)$ where $k$ gives the number of possible values of price for which the expected profit $\operatorname{EP}\left(Q_{1}, p, i\right)$ is computed in Step 2 in Algorithm 2. By decreasing the grid width parameter $\Delta p$, we can improve the accuracy of the result. Similarly, $\Delta Q$ is used to discretize the search region for $Q_{1}$.

Algorithm 2 (Optimal solution-stochastic demand). Step 0 : Set $Q_{1}=0$, maxprofit $=0$, the state index $i=0$.

Step 1: Set $p=p^{1}, i=i+1, \operatorname{profit}\left(Q_{1}, i\right)=0$.

Step 2: Set $p=p+\Delta p$. Calculate $Q_{2 i}$ and the retailer's expected profit $E P\left(Q_{1}, p, i\right)$ by assuming $n=1$, and the purchase cost at time 2 is $c_{2 \mathrm{i}}$. If $E P\left(Q_{1}, p, i\right)>\operatorname{profit}\left(Q_{1}, i\right)$, set $\operatorname{profit}\left(Q_{1}, i\right)=E P\left(Q_{1}, p, i\right)$, and $p_{i}^{\prime}=p$. If $E P\left(Q_{1}, p, i\right) \leq$ profit $\left(Q_{1}, i\right)$, take no action. Repeat this step while $p \leq p^{u}$.

Step 3: If $i<n$, go to Step 1. Otherwise, go to Step 4 .
Step 4: Calculate the retailer's expected profit $B\left(Q_{1}\right)=$ $\sum_{i=1}^{n} w_{i}$ profit $\left(Q_{1}, i\right)$. If $B\left(Q_{1}\right)>$ maxprofit, set maxprofit $=$ $B\left(Q_{1}\right), Q_{1}^{*}=Q_{1}, p_{j}^{*}=p_{j}^{\prime}$ (for $j=1$ to $n$ ). If $B\left(Q_{1}\right) \leq$ maxprofit, go to Step 5 .

Step 5: Set $Q_{1}=Q_{1}+\Delta Q$ and set the state index $i=0$. If $Q_{1} \leq Q_{1}^{u}$, go to Step 1 . Otherwise, stop.

In Step 2, the expected profit in the two-stage, singlestate problem associated with a given pair of initial order $Q_{1}$ and price $p$ is referred to as $\operatorname{EP}\left(Q_{1}, p, i\right)$. Note that as different from Algorithm 1, for finding $Q_{1}^{*}$ we calculate the expected profit for all possible values of $Q_{1}$ between 0 and $Q_{1}^{u}$. In Algorithm 1 we stop the search when expected profit starts to decrease as we increase $Q_{1}$.

Algorithm 2 can also be used if the deterministic part of the demand function $a-b p$ is replaced by any other function of $p$. Some researchers have assumed that the random demand $Y$ depends on a multiplicative error term: $Y(p, \zeta)=y(p) \zeta$, where $y(p)$ is the deterministic part of the demand. The solution procedure for the additive error model can be adapted to the multiplicative error case as long as $y(p)$ is assumed to be known. In the multiplicative error model, the variance of demand decreases as price increases. Given a selling price $p$, the mean demand is $y(p) \mu_{e}$ and the demand variance is $[y(p)]^{2} \sigma_{e}{ }^{2}$. To use Algorithm 2 in the multiplicative error case, we obtain the posterior distribution of $\mu_{e}$ from the market signal in a manner similar to that in the additive error case. We calculate the mean and standard deviation of the predictive demand distribution at time 2 by $y(p)$ $\mu_{2}$ and $y(p) \sigma_{2}$, respectively. Hence we use these price-dependent values for demand mean and demand standard deviation in calculating the retailer's expected profit $B\left(Q_{1}\right)$.

\section{Order-cancellation flexibility}

In some cases, at time 2 it may be possible for the retailer to cancel all or part of his order previously committed at time 1 . However, the refund per unit $r$ given by the supplier for the cancelled units may be less than the price paid at time $1, c_{1}$. Order-cancellation flexibility was incorporated into a two-period model with a constant purchase price in the second period in Milner and Rosenblatt (2002). In a two-stage purchase contract, Huang et al. (2005) assume that order adjustment at time 2 incurs both a fixed and a variable cost. However in our study, purchase price at time 2 is assumed to be uncertain. In this section we first determine the optimal ordering policy under order-cancellation option given the fixed selling price, and subsequently discuss the solution procedure in the more complicated case of joint ordering and pricing problem.

\subsection{Fixed selling price}

We first consider the case of a fixed selling price; later we will discuss the case of price-sensitive demand. We assume that before placing the initial order at time 1 , the retailer and the supplier agree that the retailer will be 
refunded $r$ for each unit cancelled at time 2. Alternatively, it can be thought that at time 1 the retailer buys $Q_{1}$ call options which give him the right to order up to $Q_{1}$ units at a predetermined unit purchase (exercise) cost of $r$ at time 2 ; the cost of the option is $c_{1}-r$ per unit.

In order to solve the retailer's problem in this new scenario, we begin by considering the retailer's decision at time 2 . After observing the purchase price $c_{2 i}$ at time 2 , the retailer has the option of cancelling any portion of the initial order $Q_{1}$. Regardless of the demand signal received between time 1 and time 2, the retailer will cancel all of $Q_{1}$ if the purchase cost realized at time $2 c_{2 i}$ is less than or equal to the refund $r$; all cancelled units can be easily replaced by the cheaper units purchased at time 2 . If $c_{2 i} \geq r$, the retailer will decide the new stocking level based on the market information obtained; the new stocking level can be below or above $Q_{1}$. Let $Q$ be the total inventory that the retailer has after cancellation or additional ordering at time 2 . When $c_{2 i} \leq r$, the retailer's expected profit will be

$B_{2 i}\left(Q_{1}, Q\right)=r Q_{1}-c_{2 i} Q+p E[\min (Q, Y)]-h E[Q-Y]^{+}$.

Using the first-order condition, the optimal order quantity at time $2, Q^{*}$, is given by

$Q^{*}=\mu_{2}+\left(d_{2}+\sigma_{1}^{2}\right)^{0.5} \Phi^{-1}\left(s_{i}\right)$.

Thus, when $c_{2 i} \leq r$, the retailer's expected profit at time 2

$$
\begin{aligned}
A_{1 i}\left(Q_{1}, \mu_{2}\right)= & r Q_{1}+\left(p-c_{2 i}\right) \mu_{2}-\left(h+c_{2 i}\right)\left(d_{2}+\sigma_{1}^{2}\right)^{0.5} \Phi^{-1}\left(s_{i}\right) \\
& -(p+h)\left(d_{2}+\sigma_{1}^{2}\right)^{0.5} \Psi\left(\Phi^{-1}\left(s_{i}\right)\right) .
\end{aligned}
$$

Let $Q_{p i}$ equal the RHS of (16). We now consider the states where $c_{2 i}>r$. When $c_{2 i}>r$, the retailer's expected profit function will be different depending on whether $Q \geq Q_{1}$, or $Q<Q_{1}$. When $Q \geq Q_{1}$, the retailer adds $\left(Q-Q_{1}\right)$ units to inventory at time 2 , and we have

$B_{2 i}\left(Q_{1}, Q\right)=-c_{2 i}\left(Q-Q_{1}\right)+p E[\min (Q, Y)]-h E[Q-Y]^{+}$.

The value of $Q$ maximizing (17) is given by

$Q^{*}=\max \left\{Q_{1}, \mu_{2}+\left(d_{2}+\sigma_{1}^{2}\right)^{0.5} \Phi^{-1}\left(s_{i}\right)\right\}$.

If $Q<Q_{1}$, it means the retailer cancels $\left(Q_{1}-Q\right)$ units, and its profit function is written as

$B_{2}\left(Q_{1}, Q\right)=r\left(Q_{1}-Q\right)+p E[\min (Q, Y)]-h E[Q-Y]^{+}$,

which is maximized by

$Q^{*}=\mu^{2}+\left(d_{2}+\sigma_{1}^{2}\right)^{0.5} \Phi^{-1}[(p-r) /(p+h)]$.

Let $Q_{m i}$ equal the RHS of (20). Note that when $c_{2 i}>r$, (16) and (20) imply that $Q_{m i} \geq Q_{p i}$. Combining results for the cases $Q \geq Q_{1}$ and $Q<Q_{1}$, the retailer's optimal policy when $c_{2 i}>r$ is

$Q^{*}=\left[\begin{array}{ll}Q_{p i} & \text { if } Q_{1}<Q_{p i}, \\ Q_{1} & \text { if } Q_{p i} \leq Q_{1} \leq Q_{m i}, \\ Q_{m i} & \text { if } Q_{1}>Q_{m i} .\end{array}\right.$

Thus, in state $i$ at time 2 the retailer compares the initial order $Q_{1}$ with the two state-dependent thresholds $Q_{p i}$ and $Q_{m i}$. If $Q_{1}<Q_{p i},\left(Q_{p i}-Q_{1}\right)$ units are ordered. If $Q_{1}>Q_{m i}$,
$\left(Q_{1}-Q_{m i}\right)$ units are cancelled. If $Q_{1}$ is between the threshold values, no ordering or cancelling is made at time 2 . Observe from (16) and (20) that as the demand signal $x$ increases, the mean of the predictive distribution $\mu_{2}$ increases, which leads to an increase in $Q_{p i}$ and $Q_{m i}$.

In light of the retailer's optimal policy, we now write the retailer's expected profit expressions in the states where $c_{2 i}>r$. If $Q_{1}<Q_{p i}$, i.e., $\mu_{2}>Q_{1}-\left(d_{2}+\sigma_{1}^{2}\right)^{0.5} \Phi^{-1}\left(s_{i}\right)$,

$$
\begin{aligned}
A_{2 i}\left(Q_{1}, \mu_{2}\right)= & \left(p-c_{2 i}\right) \mu_{2}-\left(h+c_{2 i}\right)\left(d_{2}+\sigma_{1}^{2}\right)^{0.5} \Phi^{-1}\left(s_{i}\right) \\
& -(p+h)\left(d_{2}+\sigma_{1}^{2}\right)^{0.5} \Psi\left(\Phi^{-1}\left(s_{i}\right)\right)+c_{2 i} Q_{1} .
\end{aligned}
$$

Define $\quad s_{r}=(p-r) /(p+h) . \quad$ If $\quad Q_{1}-\left(d_{2}+\sigma_{1}^{2}\right)^{0.5} \quad \Phi^{-1}$ $\left(s_{r}\right) \leq \mu_{2} \leq Q_{1}-\left(d_{2}+\sigma_{1}^{2}\right)^{0.5} \Phi^{-1}\left(s_{i}\right)$,

$$
\begin{aligned}
A_{3}\left(Q_{1}, \mu_{2}\right)= & p \mu_{2}+h\left(\mu_{2}-Q_{1}\right) \\
& -(p+h)\left(d_{2}+\sigma_{1}^{2}\right)^{0.5} \Psi\left[\left(Q_{1}-\mu_{2}\right) /\left(d_{2}+\sigma_{1}^{2}\right)^{0.5}\right] .
\end{aligned}
$$

And finally, if $\mu_{2}<Q_{1}-\left(d_{2}+\sigma_{1}^{2}\right)^{0.5} \Phi^{-1}\left(s_{r}\right)$,

$$
\begin{aligned}
A_{4}\left(Q_{1}, \mu_{2}\right)= & r\left[Q_{1}-\mu_{2}-\left(d_{2}+\sigma_{1}^{2}\right)^{0.5} \Phi^{-1}\left(s_{r}\right)\right] \\
& +p \mu_{2}-h\left(d_{2}+\sigma_{1}^{2}\right)^{0.5} \Phi^{-1}\left(s_{r}\right) \\
& -(p+h)\left(d_{2}+\sigma_{1}^{2}\right)^{0.5} \Psi\left[\Phi^{-1}\left(s_{r}\right)\right] .
\end{aligned}
$$

We now combine the results for all states to derive the expected profit function at time 1 . Define the sets $V_{1}=\left\{i: c_{2 i} \leq r\right\}$, and $V_{2}=\left\{i: c_{2 i}>r\right\}$. The retailer's expected profit at time $1, B_{1}$, can be expressed as

$$
\begin{aligned}
B_{1}\left(Q_{1}\right)= & \sum_{i \in V_{1}} w_{i} \int_{-\infty}^{\infty} A_{1 i}\left(Q_{1}, \mu_{2}\right) g\left(\mu_{2}\right) d \mu_{2} \\
& +\sum_{i \in V_{2}} w_{i}\left\{\int_{Q_{1}-\left(d_{2}+\sigma_{1}^{2}\right)^{0.5} \Phi^{-1}\left(s_{i}\right)}^{\infty} A_{2 i}\left(Q_{1}, \mu_{2}\right) g\left(\mu_{2}\right) d \mu_{2}\right. \\
& +\int_{Q_{1}-\left(d_{2}+\sigma_{1}^{2}\right)^{0.5} \Phi^{-1}\left(s_{r}\right)}^{Q_{1}-\left(d_{2}+\sigma_{1}^{2}\right)^{0.5} \Phi^{-1}\left(s_{i}\right)} A_{3}\left(Q_{1}, \mu_{2}\right) g\left(\mu_{2}\right) d \mu_{2} \\
& \left.+\int_{-\infty}^{Q_{1}-\left(d_{2}+\sigma_{1}^{2}\right)^{0.5} \Phi^{-1}\left(s_{r}\right)} A_{4}\left(Q_{1}, \mu_{2}\right) g\left(\mu_{2}\right) d \mu_{2}\right\}-c_{1} Q_{1} .
\end{aligned}
$$

Differentiating (21), we have

$$
\begin{aligned}
\partial B_{1} / \partial Q_{1}= & \sum_{i \in V_{1}} w_{i} r+\sum_{i \in V_{2}} w_{i}\left\{c_{2 i}+\left(p-c_{2 i}\right)\right. \\
& \Phi\left(\left(Q_{1}-\left(d_{2}+\sigma_{1}^{2}\right)^{0.5} \Phi^{-1}\left(s_{i}\right)\right.\right. \\
& \left.\left.-\mu_{1}\right) /\left[d_{1}^{2} /\left(d_{1}+\sigma_{1}^{2}\right)\right]^{0.5}\right)+(r-p) \Phi\left(\left(Q_{1}\right.\right. \\
& \left.\left.-\left(d_{2}+\sigma_{1}^{2}\right)^{0.5} \Phi^{-1}\left(s_{r}\right)-\mu_{1}\right) /\left[d_{1}^{2} /\left(d_{1}+\sigma_{1}^{2}\right)\right]^{0.5}\right) \\
& -(p+h) \int_{Q_{1}-\left(d_{2}+\sigma_{1}^{2}\right)^{0.5} \Phi^{-1}\left(s_{r}\right)}^{Q_{1}-\left(d_{2}+\sigma_{2}^{2}\right)^{0.5} \Phi^{-1}\left(s_{i}\right)} \Phi\left[\left(Q_{1}-\mu_{2}\right) /\left(d_{2}+\sigma_{1}^{2}\right)^{0.5}\right] \\
& \left.\times g\left(\mu_{2}\right) d \mu_{2}\right\}-c_{1} .
\end{aligned}
$$

After some algebra, we obtain

$$
\begin{aligned}
\partial^{2} B_{1} / \partial Q_{1}^{2}= & \sum_{i \in V_{2}} w_{i}\left\{-(p+h) T_{4}\left[\Phi \left(\left(Q_{1}\right.\right.\right.\right. \\
& \left.\left.-\left(d_{2}+\sigma_{1}^{2}\right)^{0.5} \Phi^{-1}\left(s_{i}\right)-T_{2}\right) / T_{1}^{0.5}\right) \\
& \left.\left.-\Phi\left(\left(Q_{1}-\left(d_{2}+\sigma_{1}^{2}\right)^{0.5} \Phi^{-1}\left(s_{r}\right)-T_{2}\right) / T_{1}^{0.5}\right)\right]\right\},
\end{aligned}
$$


where

$T_{1}=\left[d_{1}^{2}\left(d_{2}+\sigma_{1}^{2}\right)\right] /\left[d_{1}^{2}+\left(d_{1}+\sigma_{1}^{2}\right)\left(d_{2}+\sigma_{1}^{2}\right)\right]$,

$T_{2}=\left[d_{1}^{2} Q_{1}+\mu_{1}\left(d_{1}+\sigma_{1}^{2}\right)\left(d_{2}+\sigma_{1}^{2}\right)\right] /\left[d_{1}^{2}+\left(d_{1}+\sigma_{1}^{2}\right)\left(d_{2}+\sigma_{1}^{2}\right)\right]$,

$T_{3}=\left[d_{1}^{2} Q_{1}^{2}+\mu_{1}^{2}\left(d_{1}+\sigma_{1}^{2}\right)\left(d_{2}+\sigma_{1}^{2}\right)\right] /\left[d_{1}^{2}+\left(d_{1}+\sigma_{1}^{2}\right)\left(d_{2}+\sigma_{1}^{2}\right)\right]$,

$T_{4}=\left(\left[T_{1}\left(d_{1}+\sigma_{1}^{2}\right)\right] /\left[2 \pi d_{1}^{2}\left(d_{2}+\sigma_{1}^{2}\right)\right]\right)^{0.5} \exp \left[\left(T_{2}^{2}-T_{3}\right) / 2 T_{1}\right]$.

Since $\partial^{2} B_{1} / \partial Q_{1}^{2} \leq 0, B_{1}$ is concave in $Q_{1}$, and the optimal $Q_{1}$ can be found by setting (22) to zero. We can use (3) to evaluate the integral term on the RHS of (22). The retailer's expected profit at time $1, B_{1}\left(Q_{1}\right)$, can be evaluated in a manner similar to that in the no-cancellation case. The details are in Appendix B. For completeness, the retailer's profit function under order-cancellation flexibility and deterministic demand of $\mu_{1}$ is given by

$$
\begin{aligned}
R P_{c}^{d}\left(Q_{1}\right)= & \sum_{i \in V_{1}} w_{i}\left[\left(r-c_{1}\right) Q_{1}+\left(p-c_{2 i}\right) \mu_{1}\right] \\
& +\sum_{i \in V_{2}} w_{i}\left[\left(c_{2 i}-c_{1}\right) Q_{1}+\left(p-c_{2 i}\right) \mu_{1}\right]
\end{aligned}
$$

\subsection{Price-sensitive demand}

With slight modifications, we can use Algorithms 1 and 2 to solve the retailer's problem when demand is pricedependent and order-cancellation flexibility exists. After observing the random purchase price $c_{2 i}$ at time 2 , the retailer decides how much of the initial order to cancel (if any), and how much to buy additionally. As in the problem where order cancellation is not allowed, the selling price is chosen according to the state of the world at time 2 .

If demand is deterministic, we first compare the refund $r$ and the purchase cost $c_{2 i}$ in state $i$. If $r \leq c_{2 i}$, cancelling any unit ordered at time 1 , and substituting it by a new unit purchased at price $c_{2 i}$ will decrease the retailer's profit. Hence, the retailer's optimal action in this state is same as that in the problem with no cancellation, and the retailer's profit in state $i$ is found by following the same steps for the no-cancellation case described in Algorithm 1. However, if $r>c_{2 i}$, it is also true that $c_{2 i}<c_{1}$, and the retailer can increase its profit by replacing the units ordered at time 1 by new purchases at time 2 . Hence, when $r>c_{2 i}$, the retailer cancels all of $Q_{1}$, orders $Y^{(i)}$ units at time 2 , and sets the selling price to $p_{i}$ given by (5).

To write the retailer's profit in the deterministic demand case, we divide set $V_{2}$ into two disjoint sets $V_{21}$ and $V_{22}$ which are defined by $V_{21}=\left\{i: c_{2 i}>r, Q_{1}<Y^{(i)}\right\}$ and $V_{22}=\left\{i: c_{2 i}>r, Q_{1} \geq Y^{(i)}\right\}$. Then, the retailer's profit function with order-cancellation flexibility is

$$
\begin{aligned}
R P_{c}\left(Q_{1}\right)= & -c_{1} Q_{1}+\sum_{i \in V_{1}} w_{i}\left[r Q_{1}+\left(p_{i}-c_{2 i}\right) Y^{(i)}\right] \\
& +\sum_{i \in V_{21}} w_{i}\left[p_{i} Y^{(i)}-c_{2 i}\left(Y^{(i)}-Q_{1}\right)\right]+\sum_{i \in V_{22}} w_{i}\left(\frac{a+\mu_{e}-Q_{1}}{b}\right) Q_{1} .
\end{aligned}
$$

Differentiating (25), we obtain

$$
\frac{\partial R P_{c}\left(Q_{1}\right)}{\partial Q_{1}}=-c_{1}+\sum_{i \in V_{1}} w_{i} r+\sum_{i \in V_{21}} w_{i} c_{2 i}+\sum_{i \in V_{22}} \frac{w_{i}\left(a+\mu_{e}-2 Q_{1}\right)}{b} .
$$

$R P_{c}\left(Q_{1}\right)$ is concave in $Q_{1}$ since

$\frac{\partial^{2} R P_{c}\left(Q_{1}\right)}{\partial Q_{1}^{2}}=\sum_{i \in V_{22}} \frac{-2 w_{i}}{b} \leq 0$.

Thus, when set $V_{22}$ is not empty at the optimal solution, the optimal order quantity $Q_{1}^{*}$ is given by

$Q_{1}^{*}=\frac{-b c_{1}+b \sum_{i \in V_{1}} w_{i} r+b \sum_{i \in V_{21}} w_{i} c_{2 i}+\left(a+\mu_{e}\right) \sum_{i \in V_{22}} w_{i}}{2 \sum_{i \in V_{22}} w_{i}}$.

Since set $V_{22}$ is empty when $Q_{1}=0$, we have

$\left.\frac{\partial R P_{c}\left(Q_{1}\right)}{\partial Q_{1}}\right|_{Q_{1}=0}=-c_{1}+\sum_{i=1}^{n} w_{i} \max \left(r, c_{2 i}\right)$.

Correspondingly, similar to the no-cancellation case, when $c_{1}>\sum_{i=1}^{n} w_{i} \max \left(r, c_{2 i}\right)$, the optimal solution is $Q_{1}^{*}=0, Q_{2 i}^{*}=Y^{(i)}$ and $p_{i}^{*}=p_{i}, i=1, \ldots, n$.

To integrate order-cancellation feature in the stochastic demand scenario, we can incorporate (21) into Algorithm 2. For a given selling price and $Q_{1}$, the retailer's expected profit in the single-state problem is now computed in accordance with (21) after identifying the sets $V_{1}$ and $V_{2}$ based on comparison of $r$ and $c_{2 i}$. For the states in set $V_{1}$, cancelling all of $Q_{1}$ and reordering up to the optimal stocking level $Q_{p i}$ at unit price $c_{2 i}$ is optimal. For the states in set $V_{2}$, the state-dependent policy parameters $Q_{p i}$ and $Q_{m i}$ define the retailer's optimal action at time 2. As in the no-cancellation scenario discussed in Section 5.2, $\mu_{1}$ in (21) represents the estimated error mean at time 1 , and we identify the optimal solution by grid search on $Q_{1}$ and prices.

\section{Numerical examples}

In this section we provide some numerical examples to illustrate the methodology we have developed. In the computational study, we use the following set of parameters: $h=2, \sigma_{1}^{2}=2, \mu_{1} \in\{10,15,20\}, d_{1} \in\{10,20\}$. In all examples except those in Section 7.5, the randomness in demand is considered to be additive. For the additive price-sensitive demand, we use a linear demand function $y(p)=a-b p$ with $a=30, b=1.6$. There are two possible states of the world at time 2 with probabilities $w_{1}=w_{2}=0.5$, and the purchase costs $c_{21}=4, c_{22}=7$. The unit wholesale price at time $1 c_{1}=5$. The search algorithms are implemented using $\Delta Q=\Delta p=0.1$.

\section{Table 1}

Optimal price and order quantity with additive price-sensitive demand for the single-stage problem $\left(a=30, b=1.6, c_{1}=5, h=2, \sigma_{1}^{2}=2\right)$.

\begin{tabular}{lllll}
\hline$\mu_{1}$ & $d_{1}$ & $Q_{1}(s s)$ & $P(s s)$ & $B\left(Q_{1}(s s)\right)$ \\
\hline 10 & 10 & 17.2 & 14.7 & 137.3 \\
& 20 & 17.6 & 14.5 & 129.3 \\
15 & 10 & 20.0 & 16.3 & 189.6 \\
& 20 & 20.5 & 16.2 & 181.1 \\
20 & 10 & 22.7 & 17.9 & 250.0 \\
& 20 & 23.3 & 17.8 & 240.9 \\
\hline
\end{tabular}




\subsection{Single-stage problem}

Before exploring the two-stage problem, we first present the optimal solution for the single-stage problem with stochastic demand in Table 1 ; in this scenario, we assume that the retailer orders are placed at time 1 only, and ordering at time 2 is not possible. In Table $1, p(s s)$ and $Q_{1}(s s)$ indicate the optimal price and order quantity, respectively, selected according to demand forecast at time 1 . The optimal expected profit is given by $B\left(Q_{1}(s s)\right.$ ). For a given order quantity $Q_{1}$, the optimal price $p(s s)$ in the single-stage problem satisfies the equation

$p(s s)=\left[a+\mu_{1}+b c_{1}-\Theta(z)\right] / 2 b$,

where $z=Q_{1}-[a-b p(s s)], \quad \Theta(z)=\int_{z}^{\infty}(\zeta-z) \omega(\zeta) d \zeta$, and $\omega(\zeta)$ is the pdf of the error term (Lemma 1, Petruzzi and Dada, 1999). Thus, using (30), the optimal price is expressed in terms of the order quantity. After this step, the order quantity maximizing the expected profit in the single-stage problem can be determined by conducting one-dimensional search on $Q_{1}$. The case $d_{1}=20$ compared to $d_{1}=10$ indicates a higher uncertainty in demand. The results in Table 1 imply that the optimal price (order quantity) decreases (increases) as demand uncertainty $\left(d_{1}\right)$ increases.

\subsection{Price-sensitive demand}

We now return to the two-stage problem. Implementing Algorithm 1, the optimal first-stage order $Q_{1}^{d}$ and the optimal price in state $i$ at time $2 p_{i}^{d}$ when demand is deterministic are listed in Table 2. The optimal solution

\section{Table 2}

Optimal prices and order quantities with additive price-sensitive demand for the two-stage problem $\left(a=30, b=1.6, c_{1}=5, w_{1}=0.5\right.$, $\left.w_{2}=0.5, c_{21}=4, c_{22}=7, h=2, \sigma_{1}^{2}=2\right)$.

\begin{tabular}{llllllllll}
\hline$\mu_{1}$ & $d_{1}$ & $Q_{1}^{d}$ & $R P\left(Q_{1}^{d}\right)$ & $p_{1}^{d}$ & $p_{2}^{d}$ & $Q_{1}^{s}$ & $B\left(Q_{1}^{s}\right)$ & $p_{1}^{s}$ & $p_{2}^{s}$ \\
\hline 10 & 10 & 15.2 & 160.4 & 14.5 & 15.5 & 13.0 & 145.9 & 14.3 & 15.3 \\
& 20 & 15.2 & 160.4 & 14.5 & 15.5 & 11.0 & 144.4 & 14.3 & 15.4 \\
15 & 10 & 17.7 & 214.3 & 16.1 & 17.1 & 15.5 & 198.9 & 15.9 & 16.9 \\
& 20 & 17.7 & 214.3 & 16.1 & 17.1 & 13.6 & 197.4 & 15.8 & 17.0 \\
20 & 10 & 20.2 & 276.0 & 17.6 & 18.6 & 18.1 & 259.9 & 17.4 & 18.5 \\
& 20 & 20.2 & 276.0 & 17.6 & 18.6 & 16.3 & 258.3 & 17.4 & 18.5 \\
\hline
\end{tabular}

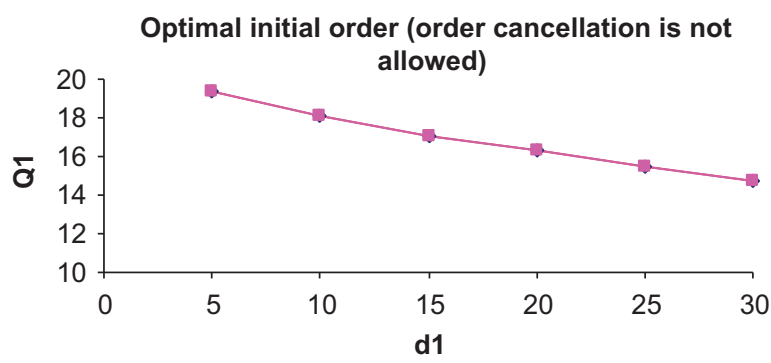

Fig. 2. Impact of demand variance on the initial order quantity when order cancellation at time 2 is not allowed $\left(a=30, b=1.6, c_{1}=5\right.$, $\left.w_{1}=0.5, w_{2}=0.5, c_{21}=4, c_{22}=7, h=2, \sigma_{1}^{2}=2, \mu_{1}=20\right)$. when demand is stochastic is described by $Q_{1}^{s}, p_{1}^{s}$, and $p_{2}^{s}$, and is obtained using Algorithm 2. It can be seen from Table 2 and Fig. 2 that the initial order $Q_{1}^{s}$ decreases as $d_{1}$ increases. Thus, higher demand uncertainty causes the retailer to reduce the first-stage order, and adopt a waitand-see strategy. On the other hand, in the single-stage problem (Table 1 ), there is a positive relationship between $\mathrm{d}_{1}$ and the order quantity $Q_{1}$.

Table 2 also lists the retailer's maximal profit under deterministic demand, $R P\left(Q_{1}^{d}\right)$, and under stochastic demand, $B\left(Q_{1}^{S}\right)$. As expected, the profit is higher when demand is known. The results indicate that the difference between the optimal prices in state 1 and state 2 is greater in the stochastic demand case compared to the deterministic demand case. In the traditional single-stage pricedependent newsvendor model with an additive error term (e.g., Petruzzi and Dada, 1999), the optimal price in the stochastic demand scenario is lower than in the deterministic demand scenario. In our model we also observe a similar pattern in both states at time 2 .

The difference in the retailer's expected profit between Tables 1 and 2 is more pronounced when demand uncertainty $\left(d_{1}\right)$ is higher. It is also observed that the order amount at time $1, Q_{1}$ decreases when a second-order opportunity exists at time 2 .

\subsection{Order cancellation (fixed price case)}

Table 3 shows the retailer's optimal order quantity and expected profit in the order-cancellation case with a fixed selling price. As expected, as the unit refund $r$ increases, the retailer's expected profit increases. The initial order quantity $Q_{1}$ is positively related to the selling price. Results in Table 3 indicate that changes in demand uncertainty have a limited effect on the retailer's expected profit. Thus, order-cancellation flexibility mitigates the negative impact of demand uncertainty on the retailer's expected profit. We note that $d_{1}$ is also a measure of expected improvement in the demand forecast. Between time 1 and time 2, the variance of demand is reduced by

Table 3

Optimal retailer policy when order cancellation is possible and selling price is fixed ( $\left.c_{1}=5, w_{1}=0.5, w_{2}=0.5, c_{21}=4, c_{22}=7, h=2, \sigma_{1}^{2}=2\right)$.

\begin{tabular}{|c|c|c|c|c|c|}
\hline$r$ & $p$ & $\mu_{1}$ & $d_{1}$ & $Q_{1}$ & $B\left(Q_{1}\right)$ \\
\hline \multirow[t]{8}{*}{3} & \multirow[t]{4}{*}{10} & \multirow[t]{2}{*}{10} & 10 & 7.2 & 39.6 \\
\hline & & & 20 & 6.1 & 38.4 \\
\hline & & \multirow[t]{2}{*}{15} & 10 & 12.2 & 64.6 \\
\hline & & & 20 & 11.1 & 63.4 \\
\hline & \multirow[t]{4}{*}{15} & \multirow[t]{2}{*}{10} & 10 & 8.1 & 85.7 \\
\hline & & & 20 & 6.9 & 84.5 \\
\hline & & \multirow[t]{2}{*}{15} & 10 & 13.1 & 135.7 \\
\hline & & & 20 & 11.9 & 134.5 \\
\hline \multirow[t]{8}{*}{4.5} & \multirow[t]{4}{*}{10} & \multirow[t]{2}{*}{10} & 10 & 10.0 & 42.4 \\
\hline & & & 20 & 10.3 & 41.5 \\
\hline & & \multirow[t]{2}{*}{15} & 10 & 15.0 & 68.6 \\
\hline & & & 20 & 15.3 & 67.7 \\
\hline & \multirow[t]{4}{*}{15} & \multirow[t]{2}{*}{10} & 10 & 10.9 & 88.7 \\
\hline & & & 20 & 11.3 & 87.8 \\
\hline & & \multirow[t]{2}{*}{15} & 10 & 15.9 & 140.0 \\
\hline & & & 20 & 16.3 & 139.1 \\
\hline
\end{tabular}


(in percentage)

$\left(\sigma_{1}^{2}+d_{1}-\sigma_{2}^{2}\right) /\left(\sigma_{1}^{2}+d_{1}\right)=d_{1}^{2} /\left(\sigma_{1}^{2}+d_{1}\right)^{2}$.

Let $w\left(d_{1}\right)=d_{1}^{2} /\left(\sigma_{1}^{2}+d_{1}\right)^{2}$. It can be shown that

$\partial w\left(d_{1}\right) / \partial d_{1}=2 d_{1} \sigma_{1}^{2} /\left(\sigma_{1}^{2}+d_{1}\right)^{3}>0$.

Thus, a higher $d_{1}$ corresponds to a greater improvement in the demand forecast as a result of new market information. Hence, a higher $d_{1}$ value implies not only a higher demand uncertainty, but also a larger reduction in demand uncertainty between time 1 and time 2 . The retailer can use the order-cancellation tool more effectively when the potential improvement in the demand forecast $\left(d_{1}\right)$ is high and demand forecasts differ significantly between time 1 and time 2 .

\subsection{Order cancellation (price-sensitive demand case)}

Numerical examples for the problem involving both price-sensitive demand and order-cancellation flexibility are reported in Table 4 . In comparing Tables 2 and 4, under stochastic demand, we see that both the retailer's expected profit and the initial order amount increase when the order-cancellation option at time 2 is allowed. If the refund is high enough, the same pattern also occurs in the deterministic demand case. The optimal prices do not change significantly whether order cancellation is possible or not. When $\mathrm{d}_{1}$ increases, the retailer's expected profit decreases slightly, and the optimal prices change negligibly. As expected, higher refund values lead to higher initial orders and expected profits.

In Tables 3 and 4, the relationship between the demand variance and the initial order quantity varies as the refund value $r$ changes. An increase in the demand variance reduces the initial order when refund is low, but the effect of the demand variance on the initial order is the opposite when refund is high. Note that as the refund value decreases, the problem with order-cancellation option becomes more similar to the problem without ordercancellation option. Hence, it is not surprising that the impact of $d_{1}$ on $Q_{1}^{s}$ in Table 2 and that in Table 4 (when $r=3$ ) are consistent. For a given refund value, Fig. 3 shows the change in the optimal initial order $Q_{1}^{s}$ in response to changes in $d_{1}$. As pointed out by a referee, a potential future research topic is to further characterize the threshold point of refund where the impact of demand variance on the initial order has a regime change.

In Tables 2 and 4, the retailer mainly adjusts the order quantity in response to changes in the demand variance, and the change in the optimal price is insignificant. These effects are similar to the impact of demand variance in the traditional single-stage problem studied in the previous research of Lau and Lau (1988). The impact of $d_{1}$ on the optimal prices $p_{1}^{s}$ and $p_{2}^{s}$ is shown in Fig. 4 (order cancellation is not allowed). We also observe that the optimal initial order quantity and price both increase as the estimated mean of the error term at time $1\left(\mu_{1}\right)$ increases.
Table 4

Optimal prices and order quantities with additive price-sensitive demand and order cancellation $\left(a=30, b=1.6, c_{1}=5, w_{1}=0.5\right.$, $\left.w_{2}=0.5, c_{21}=4, c_{22}=7, h=2, \sigma_{1}^{2}=2\right)$.

\begin{tabular}{lllllllllll}
\hline$r$ & $\mu_{1}$ & $d_{1}$ & $Q_{1}^{d}$ & $R P\left(Q_{1}^{d}\right)$ & $p_{1}^{d}$ & $p_{2}^{d}$ & $Q_{1}^{s}$ & $B\left(Q_{1}^{s}\right)$ & $p_{1}^{s}$ & $p_{2}^{s}$ \\
\hline 3 & 10 & 10 & 15.2 & 160.4 & 14.5 & 15.5 & 13.8 & 146.2 & 14.3 & 15.3 \\
& & 20 & 15.2 & 160.4 & 14.5 & 15.5 & 12.7 & 145.0 & 14.3 & 15.3 \\
& 15 & 10 & 17.7 & 214.3 & 16.1 & 17.1 & 16.4 & 199.2 & 15.9 & 16.9 \\
& & 20 & 17.7 & 214.3 & 16.1 & 17.1 & 15.3 & 198.0 & 15.9 & 16.9 \\
& 20 & 10 & 20.2 & 276.0 & 17.6 & 18.6 & 19.0 & 260.2 & 17.5 & 18.5 \\
& & 20 & 20.2 & 276.0 & 17.6 & 18.6 & 17.9 & 258.9 & 17.4 & 18.5 \\
4.5 & \multirow{2}{*}{10} & 10 & 15.6 & 164.3 & 14.5 & 15.3 & 16.8 & 150.6 & 14.3 & 15.1 \\
& & 20 & 15.6 & 164.3 & 14.5 & 15.3 & 17.3 & 149.7 & 14.3 & 15.0 \\
& 15 & 10 & 18.1 & 218.8 & 16.1 & 16.8 & 19.6 & 204.3 & 15.9 & 16.6 \\
& & 20 & 18.1 & 218.8 & 16.1 & 16.8 & 19.9 & 203.3 & 15.9 & 16.6 \\
& 20 & 10 & 20.6 & 281.1 & 17.6 & 18.4 & 22.1 & 265.9 & 17.5 & 18.2 \\
& 20 & 20.6 & 281.1 & 17.6 & 18.4 & 22.5 & 264.9 & 17.5 & 18.2 \\
\hline
\end{tabular}

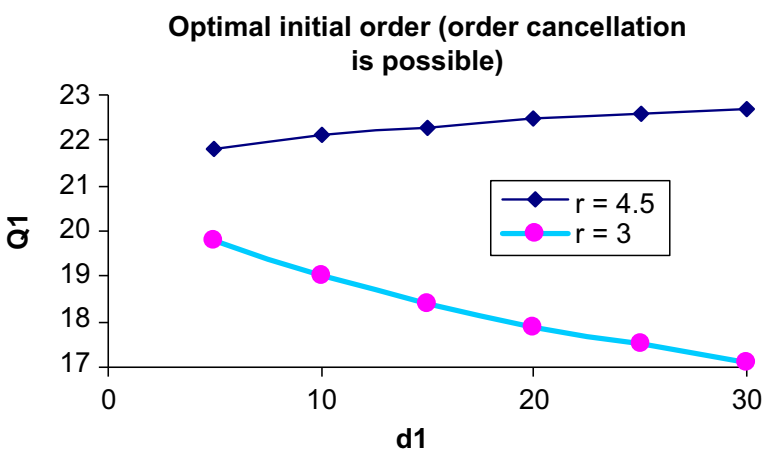

Fig. 3. Impact of demand variance and refund value on the initial order quantity when order cancellation at time 2 is allowed $(a=30, b=1.6$, $\left.c_{1}=5, w_{1}=0.5, w_{2}=0.5, c_{21}=4, c_{22}=7, h=2, \sigma_{1}^{2}=2, \mu_{1}=20\right)$.

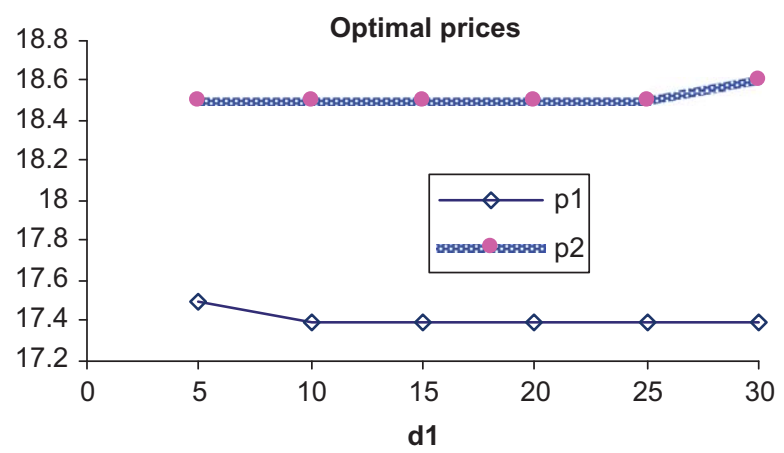

Fig. 4. Impact of demand variance on the selling prices when order cancellation at time 2 is not allowed $\left(a=30, b=1.6, c_{1}=5, w_{1}=0.5\right.$, $\left.w_{2}=0.5, c_{21}=4, c_{22}=7, h=2, \sigma_{1}^{2}=2, \mu_{1}=20\right)$.

\subsection{Multiplicative price-sensitive demand}

Finally, we present some examples for the multiplicative demand model. We use the log-linear demand function $y(p)=a p^{-b}(a>0, b>1)$, which implies that the price elasticity of demand is constant. We also use $a=1000$, $b=2, \mu_{1} \in\{3,4,5\}, d_{1} \in\{0.5,1\}, \sigma_{1}^{2}=0.25$. The remaining parameters are the same as the additive error scenario. 
Table 5

Optimal prices and order quantities with multiplicative price-sensitive demand for the two-stage problem $\left(a=1000, b=2, c_{1}=5, w_{1}=0.5\right.$, $\left.w_{2}=0.5, c_{21}=4, c_{22}=7, h=2, \sigma_{1}^{2}=0.25\right)$.

\begin{tabular}{llllll}
\hline$\mu_{1}$ & $d_{1}$ & $Q_{1}^{s}$ & $B\left(Q_{1}^{s}\right)$ & $p_{1}^{s}$ & $p_{2}^{s}$ \\
\hline 3 & 0.5 & 13.5 & 124.3 & 9.0 & 13.9 \\
& 1 & 11.0 & 121.9 & 9.0 & 14.4 \\
4 & 0.5 & 20.2 & 175.8 & 8.7 & 13.3 \\
& 1 & 17.3 & 173.3 & 8.7 & 13.7 \\
5 & 0.5 & 27.0 & 227.5 & 8.5 & 13.0 \\
& 1 & 24.0 & 225.0 & 8.5 & 13.3 \\
\hline
\end{tabular}

\section{Table 6}

Impact of higher error variance $d_{1}$ at time 1 on the order quantity, price and expected profit.

\begin{tabular}{lll}
\hline & $\begin{array}{l}\text { Additive linear } \\
\text { model }\end{array}$ & $\begin{array}{l}\text { Multiplicative log- } \\
\text { linear model }\end{array}$ \\
\hline $\begin{array}{l}\text { Order quantity (single-stage } \\
\text { problem) }\end{array}$ & Increases & Decreases \\
$\begin{array}{l}\text { Price (single-stage problem) } \\
\text { Expected profit (single-stage } \\
\text { problem) }\end{array}$ & $\begin{array}{l}\text { Decreases } \\
\text { Initial order } Q_{1} \text { (two-stage } \\
\text { problem) }\end{array}$ & Decreases \\
$\begin{array}{l}\text { Expected profit (two-stage } \\
\text { problem) }\end{array}$ & Decreases & Decreases \\
\hline
\end{tabular}

The optimal prices and order quantities for the multiplicative demand examples are given in Table 5. The results are similar to that in the additive error case. Higher demand variability $\left(d_{1}\right)$ results in a decrease in the initial order amount $Q_{1}$. The optimal prices do not change significantly as $\mathrm{d}_{1}$ changes. We note that when we disallow ordering at time 2 and solve the multiplicative demand examples as a single-stage problem, as different from the single-stage problem with additive linear demand in Section 7.1, we have observed that the optimal price (order quantity) increases (decreases) as demand uncertainty increases. Thus, when we employ the multiplicative log-linear demand function, the impact of demand uncertainty on the initial order is similar for the single-stage and two-stage problems. These results are summarized in Table 6.

\section{Conclusion}

The effect of demand forecast updating in a quick response environment has been analyzed based on newsvendor-like models in the literature. In this paper we have studied the optimal ordering decisions in a quick response system for a product with price-sensitive demand. The quick response system allows the retailer to update his prior beliefs about average demand level after collecting market information, and thus determine the final stocking level based on an improved demand forecast. The uncertainty in demand can be incorporated into the model either in an additive or a multiplicative fashion. The problem has also been extended to the case with an order-cancellation feature. Following the earlier research, we have assumed normally distributed demand in the Bayesian updating procedure. We have presented a practical method to compute the retailer's expected profit, thus obviating the need for simulation. The models presented in this paper can be expected to be helpful to practitioners who want to implement a two-stage ordering policy in a quick response environment, and need to choose the best price to maximize expected profit.

In the numerical examples, we have considered additive error with a linear demand function and multiplicative error with a log-linear demand function. Some conclusions from the numerical study are as follows. Changes in the variance of the error distribution have mainly caused the order quantity to change, and the impact on price has been observed to be weaker. This impact of demand variability on the optimal order quantity and price resembles that in the traditional single-stage newsvendor problem. When the optimal values of decision variables in the single-stage and twostage problems are compared, we have observed that the initial order in the two-stage problem is smaller than the order amount in the single-stage problem. In the singlestage problem with additive error, increasing demand variability causes the order amount to increase. On the contrary, in the two-stage problem, increasing demand variability leads to a decrease in the first-stage order. The benefit of using a two-stage ordering system instead of a single-stage system is higher when demand variability is higher.

In the additive error model, the behavior of the optimal price in deterministic and stochastic demand cases has a pattern similar to that seen in the traditional single-stage newsvendor problem: the optimal price in all states of the world in the stochastic demand case turns out to be less than that in the deterministic demand case. We have observed that the negative impact of demand variability on the retailer's expected profit is alleviated when the order-cancellation option is introduced. The introduction of order-cancellation flexibility also results in an increase in the initial order quantity. When order cancellation at a later time is allowed, the impact of demand variability on the initial order depends on the refund value; initial order increases with demand variance when the retailer receives a relatively high refund for the units cancelled later.

Future research may consider investigating the effect of modifying some of the modeling assumptions, for example, specifying the relationship between demand and price using different types of functions. In this paper we have considered that the retailer learns about the mean of the error distribution from the demand signal. It is also of interest to study the case where both the mean and variance of the error distribution are unknown and the demand signal is used to update the estimates of these two parameters.

\section{Acknowledgements}

The author thanks the Special Issue Editors and two anonymous reviewers for their helpful comments. 


\section{Appendix A. Calculation of the retailer's expected profit}

We first rewrite Eq. (1) as

$$
\begin{aligned}
B_{1}\left(Q_{1}\right)= & \sum_{i=1}^{n} w_{i}\left\{\int _ { \kappa _ { i } } ^ { \infty } \left[\left(p-c_{2 i}\right) \mu_{2}-\left(h+c_{2 i}\right)\left(d_{2}+\sigma_{1}^{2}\right)^{0.5} \Phi^{-1}\left(s_{i}\right)\right.\right. \\
& \left.-(p+h)\left(d_{2}+\sigma_{1}^{2}\right)^{0.5} \Psi\left(\Phi^{-1}\left(s_{i}\right)\right)+c_{2 i} Q_{1}\right] g\left(\mu_{2}\right) d \mu_{2} \\
& +\int_{-\infty}^{\kappa_{i}}\left[p \mu_{2}+h\left(\mu_{2}-Q_{1}\right)-(p+h)\left(d_{2}+\sigma_{1}^{2}\right)^{0.5}\right. \\
& \left.\left.\times \Psi\left(\left(Q_{1}-\mu_{2}\right) /\left(d_{2}+\sigma_{1}^{2}\right)^{0.5}\right)\right] g\left(\mu_{2}\right) d \mu_{2}\right\}-c_{1} Q_{1} .
\end{aligned}
$$

Using the properties of the normal distribution (e.g., Silver et al., 1998)

$K_{1 i} \equiv\left(p-c_{2 i}\right) \int_{\kappa_{i}}^{\infty} \mu_{2} g\left(\mu_{2}\right) d \mu_{2}=\left(p-c_{2 i}\right)\left[\sigma \phi\left(z_{i}\right)+\mu_{1}\left(1-\Phi\left(z_{i}\right)\right)\right]$, where $z_{i}=\left(\kappa_{i}-\mu_{1}\right) / \sigma$. We have

$$
\begin{aligned}
K_{2 i} & \equiv\left[-\left(h+c_{2 i}\right)\left(d_{2}+\sigma_{1}^{2}\right)^{0.5} \Phi^{-1}\left(s_{i}\right)+c_{2 i} Q_{1}\right] \int_{\kappa_{i}}^{\infty} g\left(\mu_{2}\right) d \mu_{2} \\
& =\left[-\left(h+c_{2 i}\right)\left(d_{2}+\sigma_{1}^{2}\right)^{0.5} \Phi^{-1}\left(s_{i}\right)+c_{2 i} Q_{1}\right]\left[1-\Phi\left(z_{i}\right)\right] .
\end{aligned}
$$

Since

$$
\begin{aligned}
\Psi(u) & =\phi(u)-u[1-\Phi(u)], \\
K_{3 i} & \equiv-(p+h)\left(d_{2}+\sigma_{1}^{2}\right)^{0.5} \Psi\left(\Phi^{-1}\left(s_{i}\right)\right) \int_{\kappa_{i}}^{\infty} g\left(\mu_{2}\right) d \mu_{2} \\
& =-(p+h)\left(d_{2}+\sigma_{1}^{2}\right)^{0.5}\left[\phi\left(\Phi^{-1}\left(s_{i}\right)\right)-\Phi^{-1}\left(s_{i}\right)+s_{i} \Phi^{-1}\left(s_{i}\right)\right]\left[1-\Phi\left(z_{i}\right)\right] .
\end{aligned}
$$

Similarly, we define

$$
\begin{aligned}
& K_{4 i} \equiv(p+h) \int_{-\infty}^{\kappa_{i}} \mu_{2} g\left(\mu_{2}\right) d \mu_{2}=(p+h)\left[\mu_{1} \Phi\left(z_{i}\right)-\sigma \phi\left(z_{i}\right)\right], \\
& K_{5 i} \equiv-h Q_{1} \int_{-\infty}^{\kappa_{i}} g\left(\mu_{2}\right) d \mu_{2}=-h Q_{1} \Phi\left(z_{i}\right) .
\end{aligned}
$$

Let

$M_{i}=-(p+h)\left(d_{2}+\sigma_{1}^{2}\right)^{0.5} \int_{-\infty}^{\kappa_{i}} \Psi\left[\left(Q_{1}-\mu_{2}\right) /\left(d_{2}+\sigma_{1}^{2}\right)^{0.5}\right] g\left(\mu_{2}\right) d \mu_{2}$.

Substituting

$\Psi\left[\left(Q_{1}-\mu_{2}\right) /\left(d_{2}+\sigma_{1}^{2}\right)^{0.5}\right]=\phi\left[\left(Q_{1}-\mu_{2}\right) /\left(d_{2}+\sigma_{1}^{2}\right)^{0.5}\right]$

$$
-\left[\left(Q_{1}-\mu_{2}\right) /\left(d_{2}+\sigma_{1}^{2}\right)^{0.5}\right]\left\{1-\Phi\left[\left(Q_{1}-\mu_{2}\right) /\left(d_{2}+\sigma_{1}^{2}\right)^{0.5}\right]\right\}
$$

and $\mu_{2}=\mu_{1}+\gamma \sigma$, we obtain

$M_{i}=K_{6 i}+K_{7 i}+K_{8 i}+K_{9 i}+K_{10 i}$,

where

$K_{6 i} \equiv-(p+h)\left(d_{2}+\sigma_{1}^{2}\right)^{0.5} \int_{-\infty}^{z_{i}} \phi\left(\frac{Q_{1}-\mu_{1}-\gamma \sigma}{\sqrt{d_{2}+\sigma_{1}^{2}}}\right) \phi(\gamma) d \gamma$,

$K_{7 i} \equiv(p+h) Q_{1} \Phi\left(z_{i}\right)$

$K_{8 i} \equiv-(p+h)\left[\mu_{1} \Phi\left(z_{i}\right)-\sigma \phi\left(z_{i}\right)\right]$,

$K_{9 i} \equiv-(p+h)\left(Q_{1}-\mu_{1}\right) \int_{-\infty}^{z_{i}} \Phi\left(\frac{Q_{1}-\mu_{1}-\gamma \sigma}{\sqrt{d_{2}+\sigma_{1}^{2}}}\right) \phi(\gamma) d \gamma$,
$K_{10 i} \equiv(p+h) \sigma \int_{-\infty}^{z_{i}} \gamma \Phi\left(\frac{Q_{1}-\mu_{1}-\gamma \sigma}{\sqrt{d_{2}+\sigma_{1}^{2}}}\right) \phi(\gamma) d \gamma$

For $K_{6 i}$ :

$$
\begin{aligned}
& \int_{-\infty}^{z_{i}} \phi\left(\frac{Q_{1}-\mu_{1}-\gamma \sigma}{\sqrt{d_{2}+\sigma_{1}^{2}}}\right) \phi(\gamma) d \gamma \\
& =\frac{1}{\sqrt{1+\beta^{2}}} \phi\left(\frac{\alpha}{\sqrt{1+\beta^{2}}}\right) \Phi\left(z_{i} \sqrt{1+\beta^{2}}+\frac{\alpha \beta}{\sqrt{1+\beta^{2}}}\right) .
\end{aligned}
$$

$K_{9 i}$ can be evaluated using (3). We can evaluate $K_{10 i}$ by

$$
\begin{aligned}
\int_{-\infty}^{z_{i}} \gamma \Phi\left(\frac{Q_{1}-\mu_{1}-\gamma \sigma}{\sqrt{d_{2}+\sigma_{1}^{2}}}\right) \phi(\gamma) d \gamma & \\
= & \frac{\beta}{\sqrt{1+\beta^{2}}} \times \phi\left(\frac{\alpha}{\sqrt{1+\beta^{2}}}\right) \Phi\left(z_{i} \sqrt{1+\beta^{2}}+\frac{\alpha \beta}{\sqrt{1+\beta^{2}}}\right) \\
& \quad-\Phi\left(\alpha+\beta z_{i}\right) \phi\left(z_{i}\right)
\end{aligned}
$$

Thus, $B_{1}\left(Q_{1}\right)$ is computed by

$B_{1}\left(Q_{1}\right)=\left(\sum_{i=1}^{n} w_{i} \sum_{j=1}^{10} K_{j i}\right)-c_{1} Q_{1}$

Hence, the retailer's expected profit can be computed in a straightforward manner by using the special functions related to the standard normal distribution, $\phi(\cdot), \Phi(\cdot)$, and $\Phi^{-1}(\cdot)$, and the cdf of standard bivariate normal distribution.

\section{Appendix B. Calculation of the retailer's expected profit in the model with order-cancellation option}

Define $z_{c}=\left[Q_{1}-\left(d_{2}+\sigma_{1}^{2}\right)^{0.5} \Phi^{-1}\left(s_{r}\right)-\mu_{1}\right] /\left[d_{1}^{2} /\left(d_{1}+\sigma_{1}^{2}\right)\right]^{0.5}$. After some algebra, we rewrite (21) as

$B_{1}\left(Q_{1}\right)=\sum_{i \in V_{1}} w_{i} D_{i}+\sum_{i \in V_{2}} w_{i}\left(\sum_{j=1}^{10} K_{j i}-\sum_{j=4}^{10} L_{j}+\sum_{j=1}^{3} N_{j}\right)-c_{1} Q_{1}$,

where

$$
\begin{aligned}
D_{i}= & r Q_{1}+\left(p-c_{2 i}\right) \mu_{1}-\left(h+c_{2 i}\right)\left(d_{2}+\sigma_{1}^{2}\right)^{0.5} \Phi^{-1}\left(s_{i}\right) \\
& -(p+h)\left(d_{2}+\sigma_{1}^{2}\right)^{0.5}\left[\phi\left(\Phi^{-1}\left(s_{i}\right)\right)-\Phi^{-1}\left(s_{i}\right)+s_{i} \Phi^{-1}\left(s_{i}\right)\right],
\end{aligned}
$$

$L_{4}=(p+h)\left[\mu_{1} \Phi\left(z_{c}\right)-\sigma \phi\left(z_{c}\right)\right]$

$L_{5}=-h Q_{1} \Phi\left(z_{c}\right)$

$L_{6}=-(p+h)\left(d_{2}+\sigma_{1}^{2}\right)^{0.5} \int_{-\infty}^{z_{c}} \phi\left(\frac{Q_{1}-\mu_{1}-\gamma \sigma}{\sqrt{d_{2}+\sigma_{1}^{2}}}\right) \phi(\gamma) d \gamma$,

$L_{7}=(p+h) Q_{1} \Phi\left(z_{c}\right)$,

$L_{8}=-(p+h)\left[\mu_{1} \Phi\left(z_{c}\right)-\sigma \phi\left(z_{c}\right)\right]$, 
$L_{9}=-(p+h)\left(Q_{1}-\mu_{1}\right) \int_{-\infty}^{z_{c}} \Phi\left(\frac{Q_{1}-\mu_{1}-\gamma \sigma}{\sqrt{d_{2}+\sigma_{1}^{2}}}\right) \phi(\gamma) d \gamma$,

$L_{10}=(p+h) \sigma \int_{-\infty}^{z_{c}} \gamma \Phi\left(\frac{Q_{1}-\mu_{1}-\gamma \sigma}{\sqrt{d_{2}+\sigma_{1}^{2}}}\right) \phi(\gamma) d \gamma$,

$N_{1}=\left\{-(p+h)\left(d_{2}+\sigma_{1}^{2}\right)^{0.5}\left[\phi\left(\Phi^{-1}\left(s_{r}\right)\right)-\Phi^{-1}\left(s_{r}\right)+s_{r} \Phi^{-1}\left(s_{r}\right)\right]\right\} \Phi\left(z_{c}\right)$,

$N_{2}=\left[r Q_{1}-(r+h)\left(d_{2}+\sigma_{1}^{2}\right)^{0.5} \Phi^{-1}\left(s_{r}\right)\right] \Phi\left(z_{c}\right)$,

$N_{3}=(p-r)\left[\mu_{1} \Phi\left(z_{c}\right)-\sigma \phi\left(z_{c}\right)\right]$.

The terms $L_{6}, L_{9}$, and $L_{10}$ can be evaluated similar to their counterparts $K_{6 i}, K_{9 i}$, and $K_{10 i}$ defined in Appendix A.

\section{References}

Bitran, G.R., Haas, E.A., Matsuo, H., 1986. Production planning of style goods with high setup costs and forecast revisions. Operations Research 34, 226-236.

Bradford, J.W., Sugrue, P.K., 1990. A Bayesian approach to the two-period style-goods inventory problem with single replenishment and heterogeneous Poisson demands. Journal of the Operational Research Society 41, 211-218.

Cattani, K.D., Dahan, E., Schmidt, G.M., 2008. Tailored capacity: speculative and reactive fabrication of fashion goods. International Journal of Production Economics 114, 416-430.

Chen, H., Chen, J., Chen, Y., 2006. A coordination mechanism for a supply chain with demand information updating. International Journal of Production Economics 103, 347-361.

Choi, T.M., 2007. Pre-season stocking and pricing decisions for fashion retailers with multiple information updating. International Journal of Production Economics 106, 146-170.

Choi, T.M., Chow, P-S., 2008. Mean-variance analysis of quick response program. International Journal of Production Economics 114, 456-475.

Choi, T.M., Li, D., Yan, H., 2003. Optimal two-stage ordering policy with Bayesian information updating. Journal of the Operational Research Society 54, 846-859.

Choi, T.M., Li, D., Yan, H., 2004. Optimal single ordering policy with multiple delivery modes and Bayesian information updates. Computers and Operations Research 31, 1965-1984.

Choi, T.M., Li, D., Yan, H., 2006. Quick response policy with Bayesian information updates. European Journal of Operational Research 170 788-808.

Christopher, M., Lowson, R., Peck, H., 2004. Creating agile supply chains in the fashion industry. International Journal of Retail and Distribution Management 32, 367-376.

Donohue, K.L., 2000. Efficient supply contracts for fashion goods with forecast updating and two production modes. Management Science 46, 1397-1411.

Eppen, G.D., Iyer, A.V., 1997. Backup agreements in fashion buying-the value of upstream flexibility. Management Science 43, 1469-1484

Federgruen, A., Heching, A., 1999. Combined pricing and inventory control under uncertainty. Operations Research 47, 454-475.

Ferguson, M.E., DeCroix, G.A., Zipkin, P.H., 2005. Commitment decisions with partial information updating. Naval Research Logistics 52, 780-795.
Fisher, M., Rajaram, K., Raman, A., 2001. Optimizing inventory replenishment of retail fashion products. Manufacturing and Service Operations Management 3, 230-241.

Fisher, M., Raman, A., 1996. Reducing the cost of demand uncertainty through accurate response to early sales. Operations Research 44 87-99.

Gurnani, H., Tang, C.S., 1999. Optimal ordering decisions with uncertain cost and demand forecast updating. Management Science 45 1456-1462.

Huang, H., Sethi, S.P., Yan, H., 2005. Purchase contract management with demand forecast updates. IIE Transactions 37, 775-785.

Iyer, A.V., Bergen, M.E., 1997. Quick response in manufacturer-retaile channels. Management Science 43, 559-570.

Kim, H.S., 2003. A Bayesian analysis on the effect of multiple supply options in a quick response environment. Naval Research Logistics 50, 937-952.

Lau, A.H.L., Lau, H.S., 1988. The newsboy problem with price-dependent demand distribution. IIE Transactions 20, 168-175.

Lau, H.S., Lau, A.H.L., 1999. A note on Bradford-Sugrue's “A Bayesian approach to the two-period style-goods inventory problem with single replenishment and heterogeneous Poisson demands". Journal of the Operational Research Society 50, 187-188.

Milner, J.M., Rosenblatt, M.J., 2002. Flexible supply contracts for short life-cycle goods: the buyer's perspective. Naval Research Logistics 49 25-45.

Nahmias, S., 1994. Demand estimation in lost sales inventory systems. Naval Research Logistics 41, 739-757.

Owen, D.B., 1980. A table of normal integrals. Communications in Statistics-Simulation and Computation 9, 389-419.

Perakis, G., Roels, G., 2008. Regret in the newsvendor model with partial information. Operations Research 56, 188-203.

Petruzzi, N.C., Dada, M., 1999. Pricing and the newsvendor problem: a review with extensions. Operations Research 47, 183-194.

Petruzzi, N.C., Dada, M., 2001. Information and inventory recourse for two-market, price-setting retailer. Manufacturing and Service Operations Management 3, 242-263.

Ray, S., Gerchak, Y., Jewkes, E.M., 2005. Joint pricing and inventory policies for make-to-stock products with deterministic pricesensitive demand. International Journal of Production Economics 97, 143-158.

Serel, D.A., 2008. Inventory and pricing decisions in a single-period problem involving risky supply. International Journal of Production Economics 116, 115-128.

Silver, E.A., Pyke, D.F., Peterson, R., 1998. Inventory Management and Production Planning and Scheduling, third ed. Wiley, New York.

Tang, C.S., Rajaram, K., Alptekinoglu, A., Ou, J., 2004. The benefits of advance booking discount programs: model and analysis. Management Science 50, 465-478.

Teng, J.T., Chang, C.T., Goyal, S.K., 2005. Optimal pricing and ordering policy under permissible delay in payments. International Journal of Production Economics 97, 121-129.

Venkateswaran, J., Son, Y.J., 2007. Effect of information update frequency on the stability of production-inventory control systems. International Journal of Production Economics 106, 171-190.

Wu, Y.N., Edwin Cheng, T.C., 2008. The impact of information sharing in a multiple-echelon supply chain. International Journal of Production Economics 115, 1-11.

Yan, H., Liu, K., Hsu, A., 2003. Optimal ordering in a dual-supplier system with demand forecast updates. Production and Operations Management 12, 30-45.

Yan, H., Tang, S.L., Yen, G., 2008. Quick response procurement cost control strategy for fabric manufacturing. International Journal of Production Research 46, 4769-4784. 